\title{
Hyperphosphorylated tau self-assembles into amorphous aggregates eliciting TLR4-dependent inflammatory responses
}

\section{David Klenerman ( $\nabla$ dk10012@cam.ac.uk)}

University of Cambridge https://orcid.org/0000-0001-7116-6954

Jonathan Meng

University of Cambridge https://orcid.org/0000-0002-4315-3483

\section{Yu Zhang}

University of Cambridge

Dominik Saman

University of Oxford https://orcid.org/0000-0002-3168-8544

\section{Suman De}

University of Cambridge https://orcid.org/0000-0003-1675-0773

\section{Jason Sang}

University of Cambridge

Kun Jiang

University of Cambridge

Jane Humphrey

University of Cambridge https://orcid.org/0000-0002-6825-1426

\section{Linda Julian}

University of Cambridge

\section{Eric Hidari}

University of Cambridge https://orcid.org/0000-0002-4777-5239

\section{R. Andres Floto}

University of Cambridge

\section{Steven Lee}

University of Cambridge

\section{Clare Bryant}

University of Cambridge https://orcid.org/0000-0002-2924-0038

Justin Benesch

University of Oxford https://orcid.org/0000-0002-1507-3742

Yu Ye

University of Cambridge https://orcid.org/0000-0003-0441-6399 


\section{Article}

Keywords: inflammatory responses, aggregates, proteins

Posted Date: April 15th, 2021

DOI: https://doi.org/10.21203/rs.3.rs-374549/v1

License: (c) (i) This work is licensed under a Creative Commons Attribution 4.0 International License. Read Full License

Version of Record: A version of this preprint was published at Nature Communications on May 16th, 2022. See the published version at https://doi.org/10.1038/s41467-022-30461-x. 


\title{
Hyperphosphorylated tau self-assembles into amorphous aggregates eliciting TLR4-dependent inflammatory responses
}

\section{Author list}

Jonathan X. Meng ${ }^{1,4, \dagger}$, Yu Zhang ${ }^{1,2,3, \dagger}$, Dominik Saman ${ }^{5}$, Suman De ${ }^{1,6}$, Jason C. Sang ${ }^{1,4}$, Kun Jiang $^{1}$, Jane Humphrey ${ }^{1}$, Linda Julian ${ }^{1,7}$, Eric Hidari ${ }^{4}$, R. Andres Floto ${ }^{2,3}$, Steven F. Lee ${ }^{1}$, Clare E. Bryant ${ }^{8}$, Justin L.P. Benesch ${ }^{5}$, Yu Ye ${ }^{1,9,10}$, David Klenerman ${ }^{1,4, *}$

\section{Affiliation}

1. Department of Chemistry, University of Cambridge, UK.

2. Molecular Immunity Unit, Department of Medicine, MRC Laboratory of Molecular Biology, University of Cambridge, UK.

3. Cambridge Centre for AI in Medicine, University of Cambridge, UK.

4. UK Dementia Research Institute at Cambridge, UK

5. Department of Chemistry, University of Oxford, UK.

6. Department of Neuroscience SITraN, University of Sheffield, UK

7. Cancer Research UK Cambridge Institute, University of Cambridge, UK

8. Medicine and Veterinary Medicine, University of Cambridge, UK

9. Department of Brain Sciences, Imperial College London, UK

10. UK Dementia Research Institute at Imperial College London, UK

$\dagger$ co-first authors

* corresponding author dk10012@cam.ac.uk

\begin{abstract}
Soluble aggregates of the microtubule-associated protein tau have been challenging to assemble and characterize, despite their important role in the development of tauopathies. We found that sequential hyperphosphorylation by PKA in conjugation with either GSK3- $\beta$ or SAPK4 enabled recombinant wild-type (WT) tau of isoform $0 \mathrm{~N} 4 \mathrm{R}$ to spontaneously polymerize into small amorphous aggregates in vitro. We employed tandem mass spectrometry to determine the phosphorylation sites and the degree of phosphorylation, and super-resolution microscopy and electron microscopy to characterize the morphology of aggregates formed. Functionally, in comparison with the unmodified aggregates, which require heparin induction to assemble, these self-assembled hyperphosphorylated tau aggregates more efficiently disrupt membrane bilayers and induce Toll-like receptor 4 (TLR4)-dependent inflammatory responses. Together, our results demonstrate that tau hyperphosphorylation is potentially damaging to cells, providing a mechanistic model of how hyperphosphorylation of tau aggregates drives neuroinflammation in tauopathies.
\end{abstract}




\section{Introduction}

Intracellular neurofibrillary tangles (NFTs) constituting hyperphosphorylated tau proteins are a pathological hallmark of several neurodegenerative diseases including Alzheimer's disease $(\mathrm{AD})^{1}$, Pick's disease, progressive supranuclear palsy, and corticobasal degeneration ${ }^{2}$, collectively called "tauopathies". In contrast, monomeric tau is highly soluble and intrinsically disordered, lacking significant regions of secondary structures and having long stretches of charged regions so that tau shows little intrinsic tendency for aggregation ${ }^{3}$. Thus, it is generally believed that tau proteins must undergo a sequence of biochemical and conformational changes in order to aggregate. A recent clinical study in support of this concept showed that posttranslational modifications (PTMs) occur in an ordered manner making tau prone to aggregation ${ }^{4}$.

Phosphorylation is the most common form of PTM of tau in vivo ${ }^{5}$. While the aggregation mechanism is still largely unclear, previous work has found that phosphorylation at specific sites can render tau not only better at microtubule destabilizing, therefore raising its effective concentration in the cytosol, but also more prone to aggregation ${ }^{6}$. For instance, pseudophosphorylation of tau at $\mathrm{Ser}^{202}$ and $\mathrm{Thr}^{205}$ has been demonstrated to enhance tau polymerization in vitro ${ }^{7}$. Furthermore, when tau phosphorylation is inhibited in tangle-forming transgenic mice, insoluble tau load and neurodegeneration are attenuated ${ }^{8}$. Studies using immunohistochemistry suggested that abnormal phosphorylation of tau occurs much earlier than the onset of NFTs ${ }^{9}$. Despite its importance, the identification of tau phosphorylation sites responsible for inducing the formation of neurotoxic species remains elusive, and the difficulty mainly lies in the fact that tau (0N4R) harbors up to 71 potential phosphorylation sites, all of which can be targeted by a multitude of kinases as well as phosphatases ${ }^{10}$. Early work has demonstrated that prior phosphorylation with cAMP-dependent Protein Kinase A (PKA) can prime the tau proteins for other kinases by inducing conformational changes that facilitate its further phosphorylation, affording phosphorylation sites at AD-specific epitopes ${ }^{11}$. Furthermore, a recent study that screens for the effect of 352 human kinases has shown that GSK3- $\beta$ and SAPK-4 were the most active protein kinases phosphorylating tau at $\mathrm{AD}$-specific epitopes ${ }^{12}$. Therefore, phosphorylation could potentially be regarded as a switch, turning native tau into a misfolded substrate and initiating aggregation. 
Here, we used PKA and then these two disease-relevant kinases, GSK3- $\beta$ and SAPK-4, to generate hyperphosphorylated tau with $\mathrm{AD}$-specific epitopes in vitro, as validated by our highresolution native mass spectrometry. We applied super-resolution microscopy and transmission electron microscopy to investigate the effects of hyperphosphorylation on the aggregation process of unmodified and phosphorylated tau. Our results demonstrated that hyperphosphorylated tau can spontaneously assemble into small amorphous aggregates at physiological concentrations, without the use of any external inducer. This is important since the use of external inducers like heparin have recently been cast into doubt with evidence showing that recombinant and AD-derived aggregates of tau have different conformations, properties and activities ${ }^{17}$. We found that these hyperphosphorylated tau aggregates are more cytotoxic than WT aggregates and can cause membrane permeabilization and TLR4-dependent neuroinflammation in human macrophages. Our findings together suggest that tau hyperphosphorylation produces aggregates that are cytotoxic through distinct mechanisms, offering a potential explanation for how hyperphosphorylated tau contributes to the development and progression of $\mathrm{AD}$. 


\section{Results}

\section{AD-specific epitopes on in vitro hyperphosphorylated tau}

Following a previously established protoco $^{13}$, we performed an in vitro phosphorylation assay, where recombinant WT tau of isoform 0 N4R was sequentially phosphorylated by PKA and then either GSK3- $\beta$ or SAPK4 (Fig. 1a). We examined the phosphorylation reaction by SDS-PAGE and confirmed that phosphorylated tau had a characteristic upward shift in electrophoretic mobility on SDS-PAGE compared to its non-phosphorylated standard (Fig. 1b). The extent of the band shift was dependent on the kinase involved in phosphorylation, suggesting distinct phosphorylation patterns on tau. Such differences in electrophoretic mobility can be due to different levels of phosphate incorporation and different levels of SDS-resistant conformational changes induced by phosphorylation ${ }^{14}$.

To characterize the phosphorylation sites, we digested phosphorylated tau monomers with trypsin and analyzed the corresponding tryptic peptides with liquid chromatography-tandem mass spectrometry (LC-MS/MS). Phosphorylation sites of GSK-3 $\beta$ phosphorylated tau (g-tau) and SAPK-4 phosphorylated tau (s-tau) were mapped in greater detail (SFig. 1a). Out of the 21 sites found to be phosphorylated by both kinases, our LC-MS/MS result revealed that both kinases afforded tau phosphorylation in AD-specific epitopes, including targeting sites of AT8, AT180, AT100, and PHF-1 ${ }^{15}$ (Fig. 1c).

In order to further assess the extent of phosphorylation, we employed high-resolution native mass spectrometry, which allowed us to determine the average number of phosphate groups per tau protein. The deconvoluted mass distribution for g-tau ranged between 40.0 and $42.0 \mathrm{kDa}$ with the highest peak at $40.7 \mathrm{kDa}$, which corresponded to nine phosphate groups (80 $\mathrm{Da}$ each) per tau protein (SFig. 1b). On the other hand, s-tau showed a mass distribution between 40.5 and $43.0 \mathrm{kDa}$ with its highest peak at $41.4 \mathrm{kDa}$, which was equivalent to 18 phosphate groups per tau protein (SFig. 1c). Unlike normal tau in healthy brain, which contains two to three phosphate groups, hyperphosphorylated tau in PHF derived from brain samples of AD patients harbor five to nine phosphate groups on average ${ }^{16}$. Therefore, both g-tau and s-tau can be considered as 
hyperphosphorylated. Applying a Bayesian Markov chain Monte Carlo (MCMC) algorithm, we also estimated the distributions of the number of phosphate groups per molecule for both tau species. We found that SAPK4 was more efficient than GSK3- $\beta$, not only in adding phosphate groups at more possible sites, as previously measured by our LC-MS/MS, but also incorporating more phosphate moieties per tau molecule (Fig. 1d).

\section{Hyperphosphorylated tau self-assembled to unstructured and amorphous oligomers}

Many studies have characterized the self-assembly process of hyperphosphorylated tau, suggesting that soluble phosphorylated tau can undergo spontaneous liquid-liquid phase separation and ultimately form tau aggregates under cellular conditions ${ }^{17,18}$ Therefore, both g-tau and s-tau were compared side-by-side with heparin-induced WT tau in order to examine the effect of hyperphosphorylation on the aggregation kinetics at neuronal tau concentration (2 $\mu \mathrm{M})^{19}$. The pentameric oligothiophene pFTAA with a high affinity to tau aggregates was employed to follow the evolution of aggregation in vitro ${ }^{20}$ (SFig. 2a). Fluorescence images revealed not only that both hyperphosphorylated tau species were able to form pFTAA-active aggregates by themselves, but also that such aggregations of hyperphosphorylated tau were significantly slower than that of heparin-induced WT tau, consistent with previous reports by others ${ }^{17}$. Representative images of tau aggregates at different time points are shown in Fig. 2d and SFig. 2b. To obtain quantitative insights from our TIRF images, the apparent average length of pFTAA-positive aggregates was plotted as a function of time (Fig. 2a): the heparin-induced WT tau first elongated into fibrillar aggregates with an apparent average length of $2400 \pm 500$ $\mathrm{nm}$ and then slowly fragmented into smaller aggregates, in agreement with the aggregation-andfragmentation pattern observed in our previous study ${ }^{20}$. On the other hand, g-tau and s-tau slowly polymerized into small aggregates with apparent average lengths of $530 \pm 220 \mathrm{~nm}$ and $670 \pm 400$ $\mathrm{nm}$, respectively, largely limited by diffraction. Fibrils above $1 \mu \mathrm{m}$ were observed only rarely for s-tau. Both hyperphosphorylated tau species remained as small aggregates over 400 hours of incubation.

To further probe the structural information, hyperphosphorylated tau aggregates were then prepared for super-resolution microscopy using a recent developed thioflavin-T (ThT) derivative, 
called Thioflavin-X (ThX) ${ }^{21}$ (SFig. 2a). The samples were imaged after 96 hours of incubation, the earliest time point at which self-assembled hyperphosphorylated tau and heparin-induced WT tau appeared to have distinctive structures. Based on ThX fluorescence images, the average length for WT tau was $1450 \pm 700 \mathrm{~nm}$ while g-tau and s-tau aggregates had average lengths of $190 \pm 50 \mathrm{~nm}$ and $280 \pm 90 \mathrm{~nm}$, respectively (Fig. 2b). By comparison, the filament length of WT tau was significantly larger than those formed by g-tau and s-tau while the number of aggregates was comparable among different tau species at $96 \mathrm{hr}$ time point, further corroborating our pFTAA results above (Fig. 2c). Representative ThX images of tau aggregates are shown in Fig. 2e and SFig. 3a-c. The size histograms obtained from ThX fluorescence images also revealed the morphological differences between WT tau and hyperphosphorylated tau: more than $25 \%$ of WT tau formed aggregates that were longer than $1000 \mathrm{~nm}$, whereas no g-tau and only $1.2 \%$ of s-tau were over this size threshold. On the other hand, about $99 \%$ of g-tau and $89 \%$ of s-tau aggregates stayed below a length of $500 \mathrm{~nm}$, while only half of WT tau aggregates were under that threshold (SFig. 3d).

As the intergrated fluorescence intensity from probes such as pFTAA or ThX can be influenced by the structure of tau aggregates ${ }^{20,21}$, samples of hyperphosphorylated tau aggregates were further investigated by transmission electron microscopy (TEM). Our TEM results showed that only nonfibrillar, amorphous aggregates were detected for both hyperphosphorylated species and abundant, relatively short fibrillar aggregates, for WT tau (Fig. 2f), therefore supporting our previous observations. Together, these results showed that hyperphosphorylation is sufficient to induce tau aggregation.

To estimate the extent of aggregation at $96 \mathrm{hr}$ time point, we evaluated the bands from SDSPAGE by densitometry, since high-order tau aggregates are SDS-stable and thereby unable to enter the ge ${ }^{21}$. By running various concentrations of tau aggregates and the monomer, we ensured the selected concentrations were within the linear range of our densitometric detection. Then by comparing the band intensities between aggregates and monomer, our SDS-PAGE result demonstrated that about $2 \%$ of WT tau and 1\% of g-tau and s-tau had formed SDS-stable aggregates (SFig. 4a). 


\section{Hyperphosphorylated tau aggregates effectively disrupt lipid membrane}

After characterizing the effects of hyperphosphorylation on the aggregation process, we then investigated whether such pronounced structural changes might translate to a gain of toxic function for tau. To address this question, a high-throughput calcium assay developed by our laboratory was used to measure the ability of tau aggregates to disrupt lipid membrane integrity as a proxy of tau cytotoxicity ${ }^{24}$. This approach allowed us to differentiate and quantify the membrane disruptive capability of different protein aggregates, including $\mathrm{A} \beta^{25}, \alpha$-synuclein ${ }^{26}$, $\operatorname{tau}^{27}$, as well as those in human cerebrospinal fluid from AD patients ${ }^{28}$ (Fig. 3a).

Heparin-induced WT tau aggregates and self-assembled hyperphosphorylated tau after $96 \mathrm{hr}$ of incubation along with respective monomer controls were applied at the same monomeric equivalent concentration $(100 \mathrm{nM})$ to liposomal vesicles. Percentage of calcium influx was measured as an indication of membrane permeabilization capacity for each tau species. Our results showed s-tau aggregates induced $28 \pm 6 \%$ calcium influx compared to ionomycin positive control, whereas WT tau aggregates caused $12 \pm 2 \%$ calcium influx and g-tau aggregates elicited $21 \pm 5 \%$ calcium influx in our liposomal assay (Fig. 3b). Therefore, s-tau aggregates were found to be significantly more potent than both WT tau aggregates and s-tau monomer control ( $6 \pm 5 \%$ of calcium influx $)$ at permeabilizing membrane vesicles. On the other hand, g-tau aggregates did not elicit any significant membrane disruption as compared to WT aggregates, and such functional difference between g- and s-tau might be due to their differential phosphorylation patterns and conformations.

\section{Hyperphosphorylated tau aggregates elicit inflammatory responses in human macrophages}

Apart from extracellular A $\beta$ deposits and intracellular hyperphosphorylated NFT, neuroinflammation is also a prominent pathological feature of $\mathrm{AD}^{29}$. Microglial activation was observed to be an early event prior to the formation of NFT in P301S mice ${ }^{30}$, so neuroinflammation was recently hypothesized to be not only a hallmark of AD but also a key pathological cause that accelerated the disease progression. A recent study further showed that small tau aggregates were associated with markers for neuroinflammation suggesting that tau may play a role in a feed forward mechanisms resulting in chronic neuroinflammation ${ }^{31}$. Thus, 
we wanted to investigate how different tau species affected the immune response. Another study revealed that calcium homeostasis regulates the activation of human macrophages in a highly quantitative manner ${ }^{32}$, thereby allowing us to develop a macrophage assay based on detecting intracellular calcium transients and the production of reactive oxygen species (ROS) as a sensitive measurement of macrophage activation in order to gain a better understanding of tau toxicity.

In our assay, monocyte-derived macrophage cells THP-1 were first dual-labeled with Fluo-4 AM and CellROX Deep Red to simultaneously monitor the change of cytosolic calcium concentration and ROS level in real-time (Fig. 4a). Different tau aggregate species were added to macrophages to test if they were able to cause aberrant calcium transients and stimulate ROS production as a proxy of macrophage activation. To validate our assay, we first treated our cells with lipopolysaccharides (LPS, $10 \mathrm{ng} / \mathrm{mL}$ ), and we observed vigorous calcium transients and ROS production (Fig. 4c,d). To ensure that our results were not confounded by LPS contamination of the protein aggregate during the preparation of recombinant protein from bacterial cultures, the levels of LPS quantified. (SFig. 4b). The effective concentration of endotoxin in our tau samples when treating the cells was about $0.01-0.02 \mathrm{EU} / \mathrm{mL}$, which was lower than our PBS buffer control.

To control for the variation in cell viability, signal traces were normalized to the basal intensity in each experiment and ionomycin $(1 \mu \mathrm{M})$ was perfused in the medium at the end of each trial as the positive control. Based on the results from our in vitro liposomal assay above, only s-tau aggregates taken at $96 \mathrm{hr}$ time point were used and compared with WT tau fibrils in our subsequent investigation. Our data showed s-tau aggregates at $100 \mathrm{nM}$ monomeric equivalent concentration caused strong characteristic peak-shaped calcium transients and simultaneously promoted the production of ROS shortly after the application of aggregates (Fig. 4b, SFig. 5a). As summarized in Fig. 4e, compared to WT aggregates which elicited calcium transients in $2.5 \pm$ $2 \%$ of cells, self-assembled s-tau aggregates induced calcium transients in $85 \pm 10 \%$ of the cells. The ROS level gradually increased by $18 \pm 10 \%$ when compared to the initial ROS level upon the treatment of s-tau aggregates. Application of WT tau aggregates, however, did not induce any significant changes in the ROS level (Fig. 4c,d). A similar null observation was also 
found in the s-tau monomer condition (Fig. 4c,d,). The monomer control also ensured the effect of s-tau on macrophage activation was due to the amorphous aggregates. Moreover, the s-tau monomer control consisted of not only an equivalent amount of the tau protein but also corresponding kinases and phosphorylation reaction buffer which contained a nanomolar range of ATP. The macrophage can respond to ATP in a concentration-dependent fashion by the activation of purinergic receptors, providing a mechanism to perceive local cellular damage such as necrosis which would release adenosine triphosphate into the extracellular domain. Therefore, the lack of response excluded the effect of purinergic receptors at the ATP concentration in our monomer control.

TLR4, a member of the Toll-like receptor family, has recently been demonstrated to play a crucial role in pro-inflammatory responses and ROS production triggered by $\alpha$-synuclein and A $\beta$ in immune cells ${ }^{33}$. Our previous work suggested that protofibrillar $A \beta$ aggregates can induce temporary calcium transients in astrocytes and promote the production of inflammatory species via TLR4 signaling pathway ${ }^{34}$. We therefore examined whether TLR4 was also responsible for the tau-induced proinflammatory responses observed in our macrophage assay. LPS was able to disrupt calcium homeostasis and stimulate ROS production in macrophages (Fig. 4c,d) When pre-treating the cells with Rhodobacter sphaeroides lipid A (RsLA, at $5 \mu \mathrm{g} / \mathrm{mL}$ ), a potent antagonist of LPS, this competitive inhibitor effectively mitigated LPS-induced inflammatory responses, i.e., calcium transients and ROS elevation (Fig. 4c,d, SFig. 5b). Furthermore, TAK$242(1 \mu \mathrm{M})$, another TLR4 inhibitor, also successfully moderated LPS-induced reaction: $5 \pm 2 \%$ of cells underwent calcium transients and ROS level elevated by $6 \pm 4 \%$ (Fig. 4c,d, SFig. 5c). Importantly, these TLR4 inhibitors when applied also effectively abated the macrophage activation induced by s-tau aggregates (Fig. 4e,f, SFig. 5b,c): after the treatment of RsLA, s-tau only elicited calcium transients in $4 \pm 4 \%$ of the cells and increased the ROS level by $3 \pm 2 \%$, significantly attenuated when compared to the uninhibited condition. After the treatment of TAK-242, s-tau only elicited calcium transients in $3 \pm 2 \%$ of the cells and increased the ROS level by $2.5 \pm 4 \%$. Thus, our results together suggested that the observed macrophage phagocytosis stimuli - the calcium transients and ROS elevation-was TLR4-dependent. Representative calcium and ROS traces of each condition can be found in SFig. 5 a-c. 
To investigate the proinflammatory nature of these hyperphosphorylated tau aggregates, we also measured the levels of TNF- $\alpha$ in cell culture supernatant by ELISA. As a positive control, various concentrations of LPS (varying from $10 \mathrm{pg} / \mathrm{mL}$ to $10 \mathrm{ng} / \mathrm{mL}$ ) were applied to elicit robust TLR4 activation, resulting in the release of TNF- $\alpha$ in a dose-dependent fashion (Fig. 5G). The s-tau aggregates at $500 \mathrm{nM}$ total monomer equivalent concentration induced $385 \pm 20$ $\mathrm{pg} / \mathrm{mL}$ of TNF- $\alpha$ secretion as the same concentration of WT aggregates only elicited $105 \pm 40$ $\mathrm{pg} / \mathrm{mL}$. Various concentrations of s-tau monomers did not induce a comparable level of cytokine, suggesting the aggregates as the inflammatory stimuli. The inflammatory response caused by stau aggregates was much more potent than that from the WT aggregates especially considering only about $1 \%$ of s-tau monomers formed aggregates at this time point. To further validate the mechanistic involvement of TLR4 receptor, cells were treated with TAK-242 prior to the incubation of tau aggregates. As a control, we first demonstrated that the LPS-induced secretion of TNF- $\alpha$ could be effectively attenuated by TAK-242 (Fig. 5). Our data then showed the inflammatory response to s-tau aggregates was also significantly suppressed by the TLR4 inhibitor (Fig. 5). Taken together, these data demonstrated that TLR4 receptor played a key role in mediating the proinflammatory responses elicited by hyperphosphorylated tau aggregates.

\section{Discussion}

\section{Hyperphosphorylated tau self-assembled to unstructured and amorphous aggregates}

Because monomeric tau shows little intrinsic tendency for aggregation ${ }^{3}$, external inducers, such as heparin and other polyanionic molecules have long been used to induce tau aggregation reaction in vitro ${ }^{35-37}$. Recent technological breakthroughs and developments in more sophisticated biochemical assays suggest that heparin-induced tau and AD-derived tau have distinct conformations, properties, and bioactivities ${ }^{38}$. As illustrated in Fig. 1a, heparin can stabilize the interaction between R2 and R3 by neutralizing the positively charged amino acid sequences in these two microtubule-binding domains ${ }^{38}$. In comparison, AD-derived tau aggregates rather have R3 and R4 enclosing themselves and forming the core of such pairedhelical filaments ${ }^{38}$. As these observations call the use of external aggregation inducers into question, more efforts have been focused on the role of phosphorylation in tau aggregation. 
We have produced hyperphosphorylated tau using sequential kinase treatment by PKA with either GSK3- $\beta$ or SAPK4. Our results show that both forms of hyperphosphorylated tau were able to form small, amorphous aggregates. These observations can be explained by the preexisting model under which tau self-assembles via intermolecular hydrophobic interaction, mostly mediated by microtubules-binding repeats (R1-R4), when the rest of the molecules including the basic regions flanking the repeats are neutralized by negative charges from external inducers such as heparin or phosphate groups ${ }^{39,40}$. When tau is unmodified, the acidic N- and Ctermini, as indicated by fluorescence resonance energy transfer and electron paramagnetic resonance experiments, fold over the vicinity of the microtubule-binding repeat domain, resulting in a paperclip conformation and thereby effectively preventing tau-tau intermolecular interaction $^{41}$. As demonstrated by NMR paramagnetic relaxation enhancement studies along with ensemble conformer calculations, hyperphosphorylation can cause an opening of such transient paperclip conformation ${ }^{42}$ and simultaneously stabilizes the $\alpha$-helical structures, which is associated to the aggregation process ${ }^{43}$, as pictorially illustrated in Fig. 1a. Consistent with this notion, the longest tau isoform 2N4R, having two highly acidic N-terminal inserts, effectively mitigates the basic charge of the flanking regions and therefore efficiently self-aggregate into paired helical filaments ${ }^{35}$. This inhibitory effect of the basic flanking regions can also be neutralized either by co-assembling with polyanions such as RNA, polyGlu, or heparin or by eliminating these positive fragments via truncation in vitro ${ }^{35-37,44}$. In AD and other tauopathies, hyperphosphorylation has been considered to be the key post-translational modification that neutralizes the positive chargers from these inhibitory domains, thereby enabling effective tauto-tau intermolecular hydrophobic interaction and ultimately tau polymerization ${ }^{35}$. Our observations fit well with this structural paradigm suggesting that hyperphosphorylation can induce a conformational change at the monomeric level, which ultimately renders tau prone to aggregation and more importantly these aggregates have different structures, as evidenced by the formation of aggregates with distinct morphological features. A recent structural stimulation study shows the free-energy landscapes display two channels of tau aggregation ${ }^{45}$ : with one leading to more ordered fibrils and the other to amorphous phases. The prefibrillar aggregates have more parallel in-register $\beta$-strands, which ultimately lead to forming fibrils, while the amorphous aggregates are filled with random $\beta$-strand stacking, which eventually leads to a macroscopically amorphous state. Furthermore, phosphorylation is shown to both 
thermodynamically facilitate the amorphous aggregate channel and kinetically favor the amorphous path, so this might also explain our observation in which both hyperphosphorylated tau species do not form fibrils. More importantly, a previous study identifies the formation of granular tau aggregates - similar to our observation of g-tau and s-tau — as an early sign of brain aging and $\mathrm{AD}$. Therefore, this result further bolsters the pathological relevance of our hyperphosphorylated tau synthesized in vitro ${ }^{46}$.

\section{Hyperphosphorylated tau aggregates trigger calcium influx of liposome assay suggesting toxicity via disruption of the lipid membrane}

Our structural analysis thus far indicated that hyperphosphorylation can promote tau polymerization to produce non-fibrillar aggregates. In fact, mounting evidence indicates that mature filamentous deposits are not toxic and several studies suggest that the non-fibrillar tau aggregates, an intermediate entity along the polymerization pathway, is more likely to be responsible for disease onset and progression ${ }^{47}$. Hyperphosphorylated protofibrillar tau in particular has also been implicated in memory deficits, neuronal loss, nucleocytoplasmic trafficking disruption, tau propagation and seeding both in vitro and in vivo ${ }^{48,49}$. Several mechanistic frameworks have been proposed, suggesting that early tau aggregates might disrupt membrane integrity either by nonspecific binding of amyloid oligomers to membrane surface or forming transmembrane oligomeric porelike structures, or a combination of both ${ }^{50}$. Previous studies have also indicated that such pore formation ability might be universally shared by other amyloidogenic proteins, including $\mathrm{A} \beta, \alpha$-synuclein, and prion-derived peptides besides tau, providing a more general mechanism that underlies the deleterious effects of all amyloid oligomers on lipid membrane and cell viability ${ }^{51-53}$. Others using a similar version of this liposomal permeabilization assay have demonstrated that tau protofibrils - rather than monomers or mature fibrils - can permeabilize lipid membrane better and, more importantly, cause more cell death, thereby emphasizing the biological relevance of our in vitro liposomal permeabilization assay ${ }^{54}$. Our liposome results are consistent with these previous studies demonstrating that smaller tau aggregates have a greater ability to permeabilize a lipid membrane than mature filaments. This disruption of the lipid membrane must be transient, since pore formation would lead to $100 \%$ entry of calcium ions into the liposomes. 


\section{Hyperphosphorylated tau oligomers trigger elevated calcium signal and ROS production, elicit inflammation via TLR4 receptors in human macrophages}

Immune cells from different origins, including brain-resident macrophages (microglia and astrocytes) as well as bone marrow-derived circulating monocytes, while playing an instrumental role in the clearance of protein aggregates by a variety of phagocytic and digestive mechanisms, can also participate in the neurodegenerative process by secreting proinflammatory chemicals and cytokines which exerts toxic effects on neurons ${ }^{55}$. In agreement with this notion, we observed hyperphosphorylated tau aggregates can induce calcium transients and oxidative stress in macrophage cells. Conversely, increasing evidence suggests that aberrant calcium transients and oxidative stress promote tau hyperphosphorylation and polymerization by activating numerous protein kinases while inhibiting the phosphatase activity ${ }^{56}$. Therefore, if our observation holds true for other kinases and phosphorylation events in vivo, then this imbalance between kinases and phosphates would disfavor the formation of fibrillar aggregates that are not inflammatory and at the same time shift the equilibrium to generate more amorphous hyperphosphorylated tau that elicits further inflammation.

Although the correlation between neuroinflammation and neurofibrillary tangle formation has been found in clinical data ${ }^{57}$, the causal relationship and mechanisms remain elusive. The present study further demonstrates hyperphosphorylated tau aggregates can lead to the secretion of TNF$\alpha$ - which is consistent with other in vivo studies ${ }^{58}$. When TLR4 is inhibited, we demonstrated that hyperphosphorylated tau aggregates can no longer induce macrophage activation and inflammation as indicated by calcium transients, ROS production, and cytokine release. TLR4 inhibition has also been shown to exert neuroprotective effects in neurodegeneration models ${ }^{59}$, corroborating our results and further supporting the idea that TLR4 could be a potential therapeutic target for AD.

Interestingly, our data shows a significantly higher TLR4-dependent inflammatory response to hyperphosphorylated tau aggregates with an amorphous form than to WT tau aggregates with fibrillar morphology. This difference may result from the specific binding structure required for TLR4 activation. The crystal structure of TLR4-MD2-LPS has been determined, and the study found that the degree of phosphorylation proved to be important for TLR4 recognition of LPS 
and subsequent activation, despite the fact that the TLR family employs remarkably versatile ligand recognition methods. The study demonstrated that two phosphate groups attached to the glucosamine of LPS support the formation of a stable complex by making electrostatic and hydrogen bond interactions simultaneously with the two TLRs in the complex ${ }^{60}$. Therefore, these phosphate moieties have a profound impact on the receptor-ligand interaction, and removal of either one of these phosphate groups can reduce endotoxic activity by $\sim 100$-fold. This structural insight may provide critical clues regarding the agonistic mechanisms employed by hyperphosphorylated tau aggregates that explain its inflammatory potency compared to its nonphosphorylated counterpart.

Our data shows that the calcium transients caused by tau aggregates in macrophages were TLR4dependent since the influx could be efficiently suppressed by RsLA or TAK-242. However, membrane permeabilization by tau aggregates as measured by the liposome assay could contribute to raised basal levels of $\mathrm{Ca}^{2+}$ and calcium dysregulation in neurons and glial cells and hence be an additional mechanism of toxicity in the cellular environment. Our results do not preclude the possibility in which these various mechanisms work synergistically to exert potential cellular damage. Based on our results, we propose the following model for the importance of hyperphosphorylation in tau aggregation: if hyperphosphorylated tau unlike $A \beta$ rarely forms fibrils especially during the early stages of $\mathrm{AD}$ but rather small soluble aggregates which are more toxic through various cellular and molecular mechanisms, then it will take a long time for the tau aggregates to be removed by forming large insoluble fibrils. Therefore, our results provide a possible explanation why tau aggregation is so damaging in neurodegenerative diseases.

\section{Summary}

This work systemically generated and characterized disease-relevant hyperphosphorylated tau in quantities that meet the demand for subsequent structural and toxicity characterization studies. Our results highlight the profound impact of hyperphosphorylation on the aggregation of tau leading to the spontaneous formation of small amorphous aggregates. These hyperphosphorylated tau aggregates are cytotoxic by diverse mechanisms, including membrane permeabilization and TLR4-dependent macrophage activation and inflammation, which could 
contribute to the disruption of calcium homeostasis, induction of oxidative stress, and release of proinflammatory cytokines observed in tauopathies. It therefore becomes important to now establish if cytotoxic hyperphosphorylated tau aggregates, similar to those characterized in this work, form in vivo. The mechanistic insight from this work may prove important for the development of therapeutics for AD and other tauopathies, by either preventing the formation of tau aggregates or reducing the cytotoxic effects that they cause.

\section{Acknowledgements}

We acknowledge valuable conversations with members of the Klenerman Lab and the Lee Lab.

\section{Author contribution}

J.X.M., Y.Z., C.B., Y.Y., D.K. conceived the project and designed the experiments. J.X.M. performed the hyperphosphorylation reactions and carried out the subsequent endotoxin removal procedures. L.J. performed the endotoxin quantification assay. J.X.M., Y.Z., and J.C.S. performed the imaging experiments (including diffraction-limited/super-resolution microscopy and electron microscopy) to determine the structural details of different tau aggregates. J.X.M. and E.Z. analyzed the super-resolution images using a Python script developed by E.H., J.X.M., D.S., J.L.P.B. and D.K. collaboratively designed the mass spectrometry experiments, and D.S. performed the experiments and analyzed the data. S.D. carried out the liposomal penetration assays and analyzed the data. J.X.M., Y.Z., and K.J. performed live cell imaging experiments. J.X.M., Y.Z. and J.H. analyzed the calcium/ROS imaging data using a MATLAB script developed by J.H. J.X.M., Y.Z., Y.Y., R.A.F., C.E.B., S.F.L. and D.K. interpreted the results and stewarded the overall project. J.X.M., Y.Z., C.E.B. and D.K. co-wrote the manuscript. All authors discussed the results and commented on the manuscript.

\section{Data availability}

All data and codes are available upon request.

\section{Funding and additional information}

J.X.M. is supported by Dr. Herchel Smith Fellowship from Williams College .Y.Z. is supported by the Wellcome Trust Grant 107032AIA to R.A.F., the Cambridge Cancer Center Pump Priming Grant and Royal Society University Research Fellowship to S.F.L. D.S. is supported by Clarendon Scholarship from Oxford University Press. C.E.B. is supported by a Wellcome Trust Investigator award (108045/Z/15/Z). This work is supported by the UK Dementia Research Institute which receives its funding from DRI Ltd, funded by the UK Medical Research Council, Alzheimer's Society and Alzheimer's Research UK and by the Royal Society (RP150066). 


\section{Conflict of interests}

The authors declare that they have no conflicts of interest with the contents of this article.

\section{Figures}

a

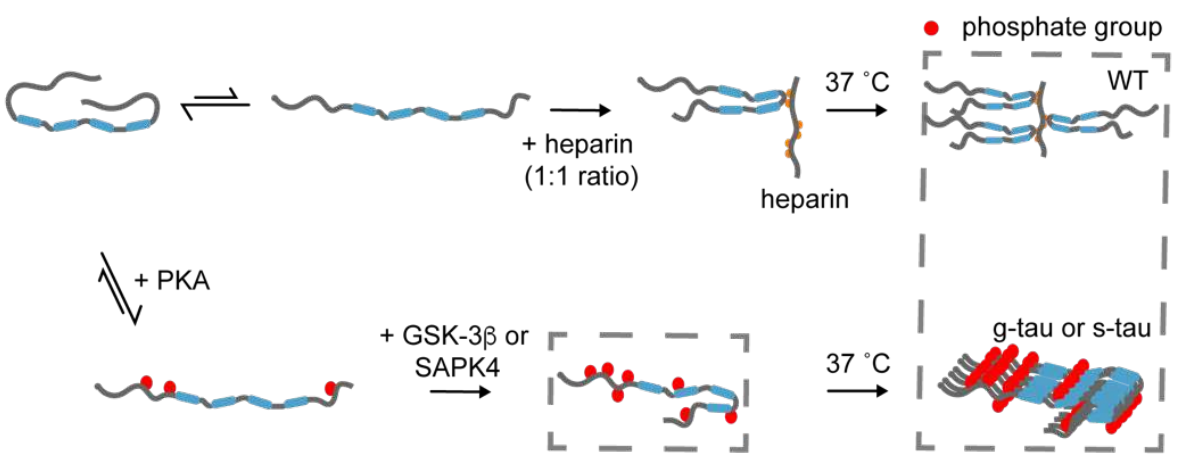

phosphorylation stablizes the monomer samples taken opening of the transient folding for mass spectrometry

aggregates taken for structural characterization via fluorescence

b

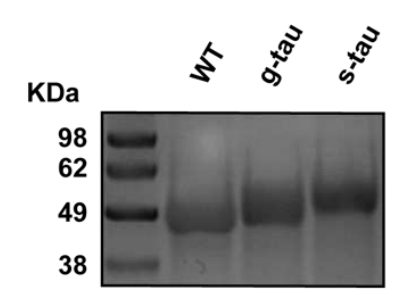

c

d microscopy
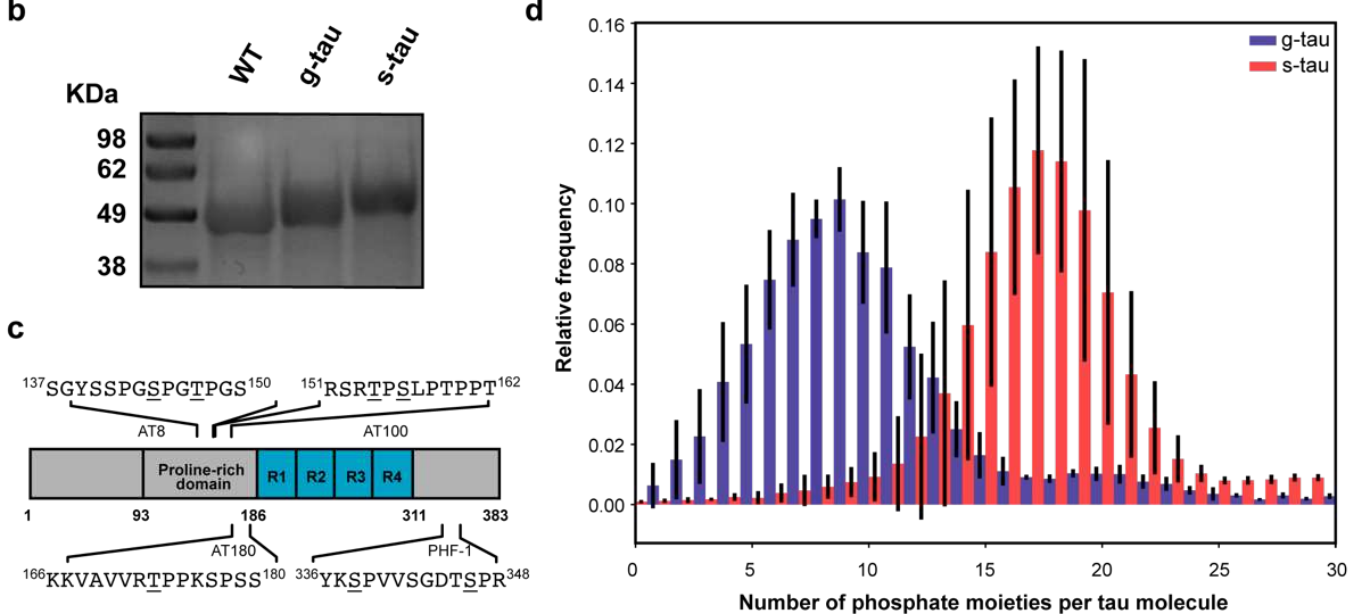

Figure 1. Sequential hyperphosphorylation of tau in vitro generates AD-specific epitopes.

a. A pictorial representation of the experimental design: WT tau was sequentially hyperphosphorylated, first by PKA and then by either GSK-3 $\beta$ or SAPK-4 kinase.

b. Representative SDS-PAGE (4-12\%, stained by Coomassie blue) showed an upward shift of electrophoretic mobility for both g-tau and s-tau species, indicating successful phosphorylation reaction.

c. LC-MS/MS analysis revealed that both g-tau and s-tau tau were hyperphosphorylated at ADspecific epitopes, such as targeting sites of AT8, AT100, AT180, and PHF-1, highlighted along with the pictorial representation of WT tau. 
d. High-resolution native mass spectrometry results indicated on average there were 9 phosphate groups per g-tau molecule while over 18 phosphate groups per s-tau molecule. Error bars represent \pm s.d. from MCMC analysis.

a
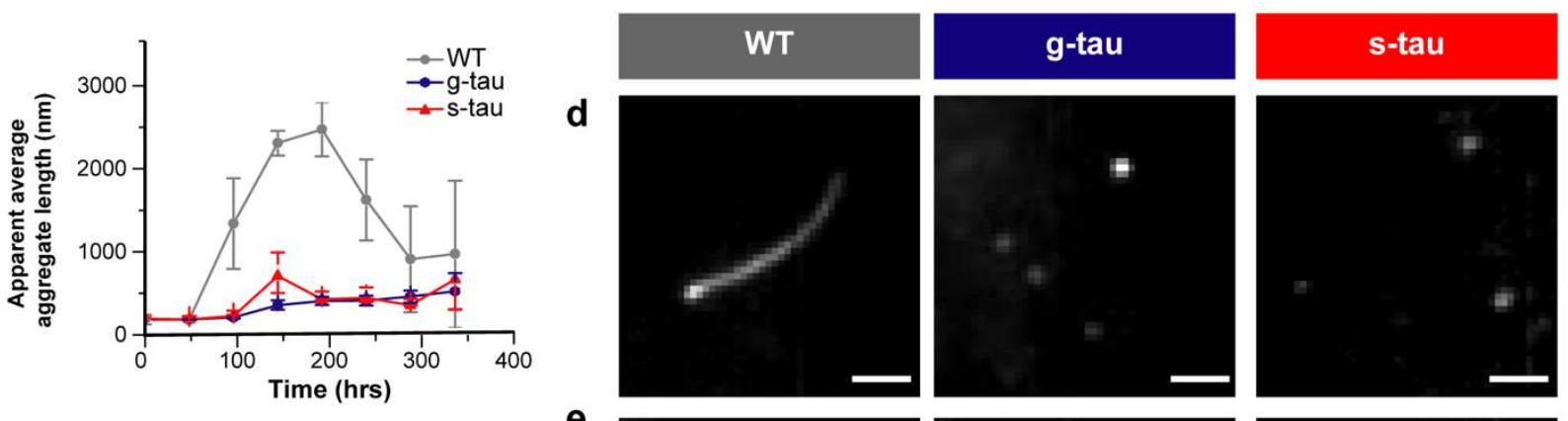

b

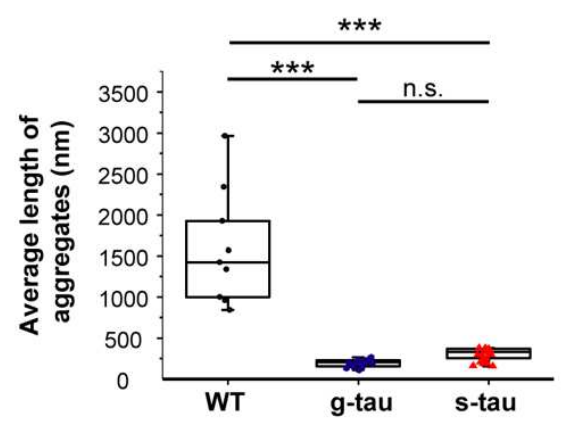

C
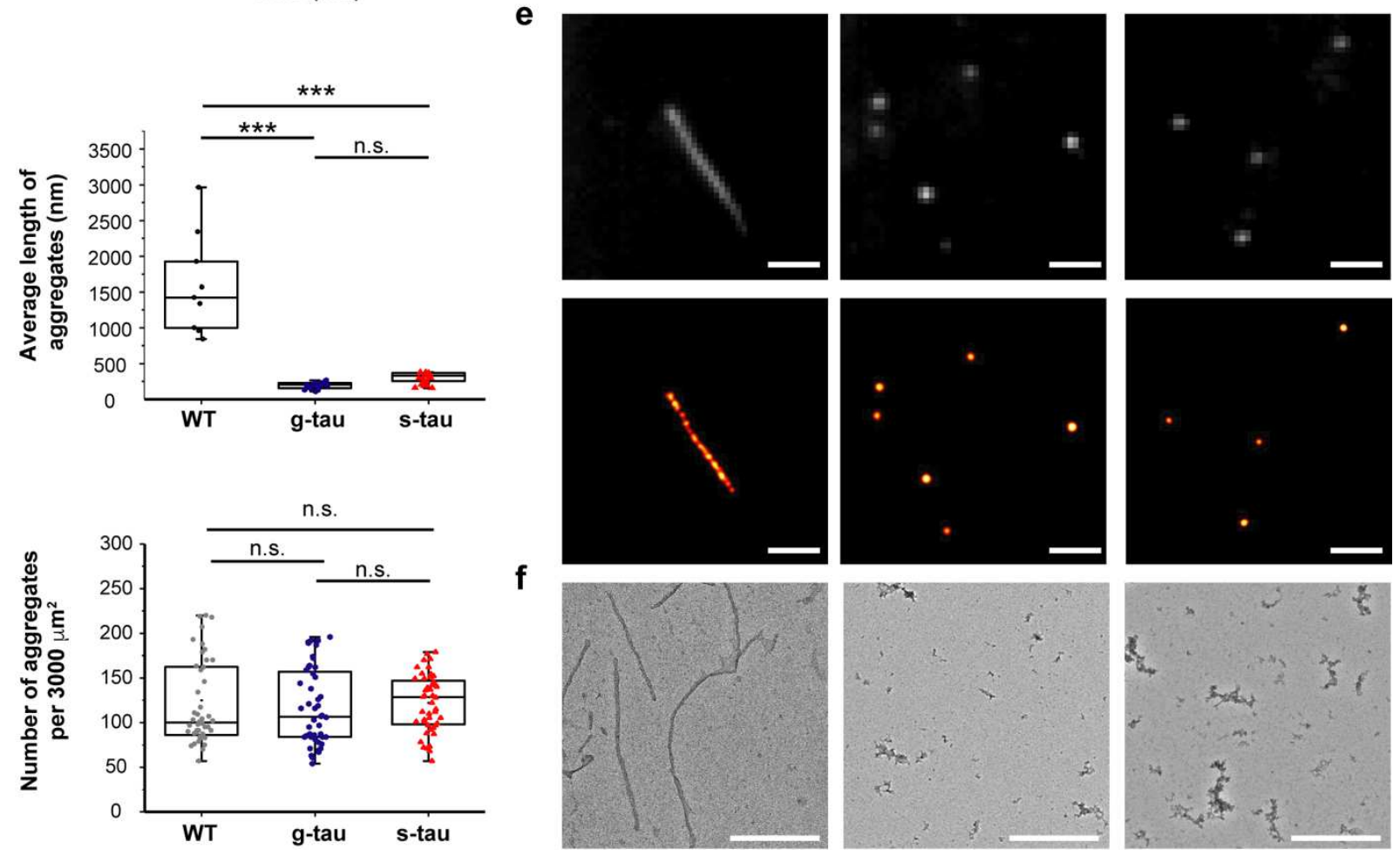

$\mathbf{f}$
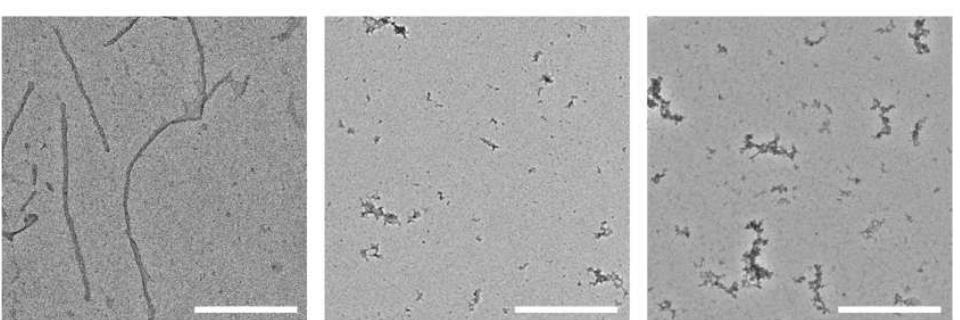

Figure 2. Hyperphosphorylation enables wildtype tau to self-polymerize into small amorphous aggregates without external inducers.

a. The apparent average length of tau aggregates, monitored by fluorescence microscopy, was analyzed and plotted as a function of time (Error bars represent \pm s.d. of $n=3$ ).

b. Summary plot based on our results from super-resolution microscopy indicated that at 96 $\mathrm{hr}$ of incubation the average length of heparin-induced WT aggregates $(1450 \pm 700 \mathrm{~nm})$ was significantly larger than both g-tau $(190 \pm 50 \mathrm{~nm})$ and or s-tau $(280 \pm 90 \mathrm{~nm})(\mathrm{n}=9$ across three independent experiments for each tau species). 
c. Summary plot based on our results from fluorescence microscopy indicated the number of aggregates was not significantly different among these three tau species ( $n=48$ across three independent experiments for each tau species).

d. Representative pFTAA diffraction-limited images of different tau species after $96 \mathrm{hr}$ of incubation: while WT tau under the induction of heparin grew into fibrillar aggregates, both g-tau and s-tau self-assembled into small, nonfibrillar aggregates which largely remained as diffraction limited spots. Scale bar: $1.0 \mu \mathrm{m}$.

e. Representative ThX stacked and super-resolved images of different tau species after $96 \mathrm{hr}$ of incubation. Scale bar: $1.0 \mu \mathrm{m}$.

f. Morphological examination of all three tau species by TEM after $96 \mathrm{hr}$ of incubation. Scale bar: $500 \mathrm{~nm}$.

For $\mathrm{b}$ and $\mathrm{c}$, the $P$ values are based on unpaired Student's t test: $* * * p<0.001$; n.s., nonsignificant.

a
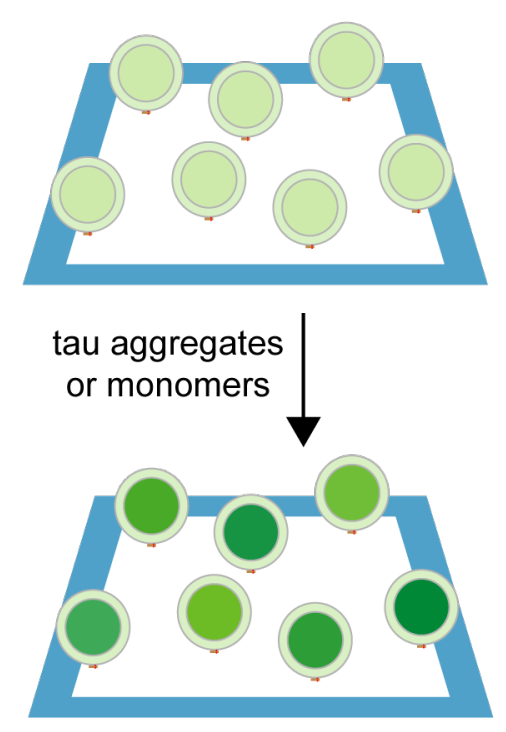

b

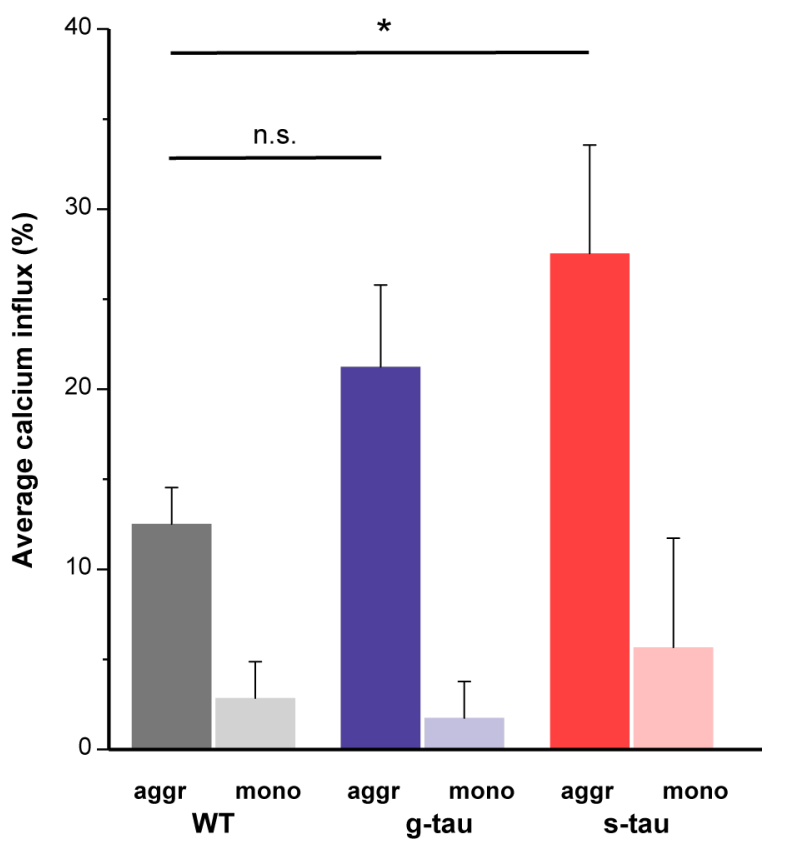

Figure 3. Hyperphosphorylated tau amorphous aggregates disrupt membrane integrity more effectively than WT fibrillar aggregates.

a. The ability to disrupt membrane integrity, an important aspect that explains the toxicity of protein aggregates, was quantified by calcium influx caused by each tau species. The percentage of $\mathrm{Ca}^{2+}$ influx was normalized by the maximum $\mathrm{Ca}^{2+}$ influx induced by ionomycin.

b. Our results from the liposomal assay revealed that WT tau aggregates caused $12 \pm 2 \%$ calcium influx, g-tau aggregates elicited $21 \pm 5 \%$ calcium influx, and s-tau aggregates induced $28 \pm 6 \%$ calcium influx. All of the monomeric controls caused less calcium 
influx than their corresponding aggregate samples. So s-tau aggregates can better permeabilize synthetic lipid bilayer than WT aggregates, suggesting a structure-function relationship of the hyperphosphorylated tau (Error bars represent \pm s.d. of $\mathrm{n}=3$ for each tau species, the $P$ values are based on unpaired Student's t test: * $p<0.05$, n.s., nonsignificant). 


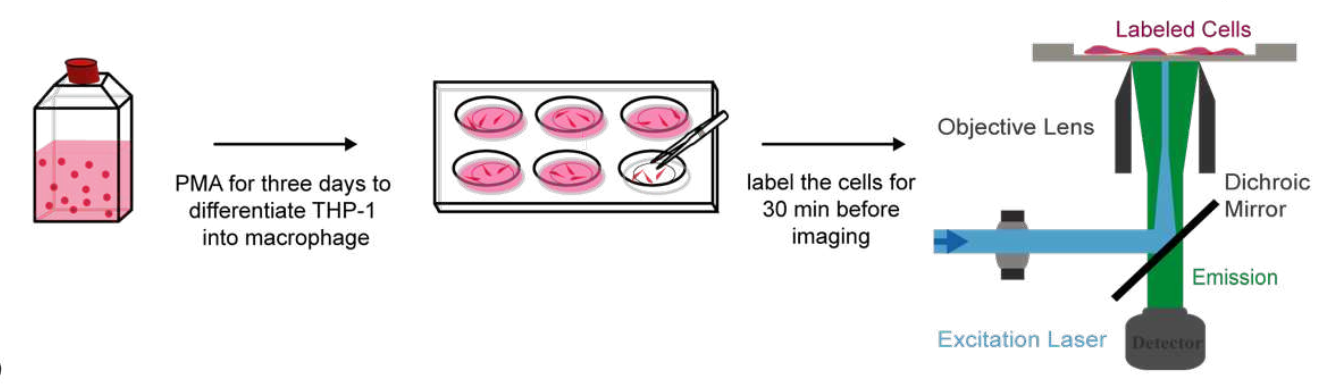

b

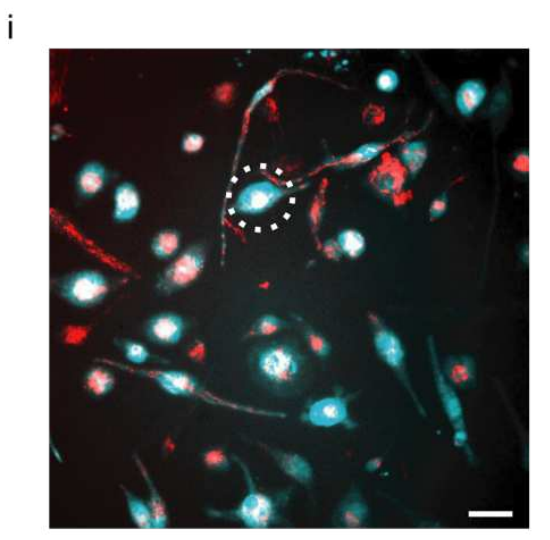

C

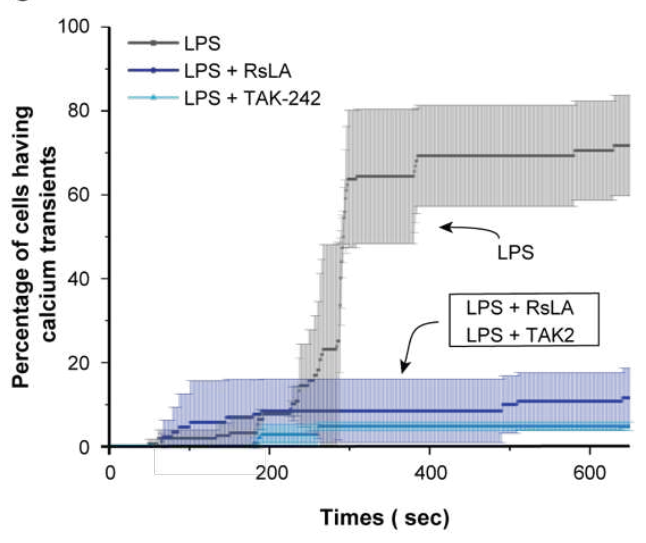

e

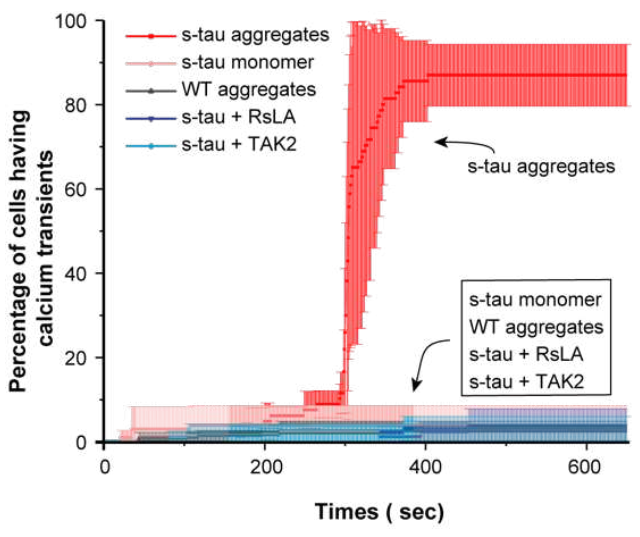

ii

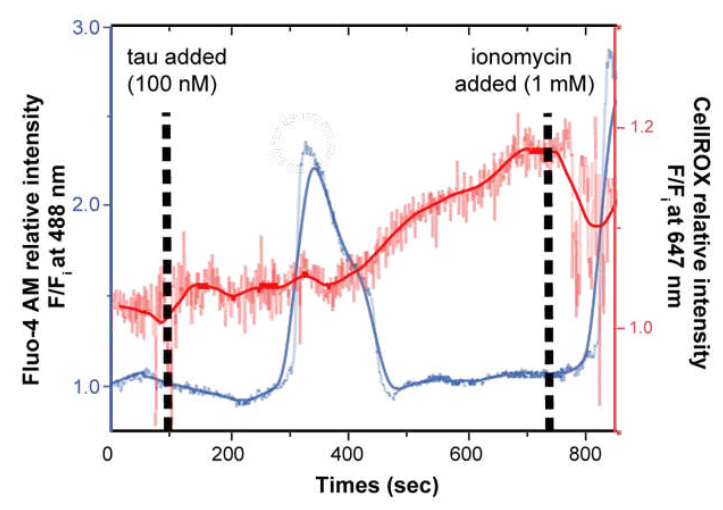

d

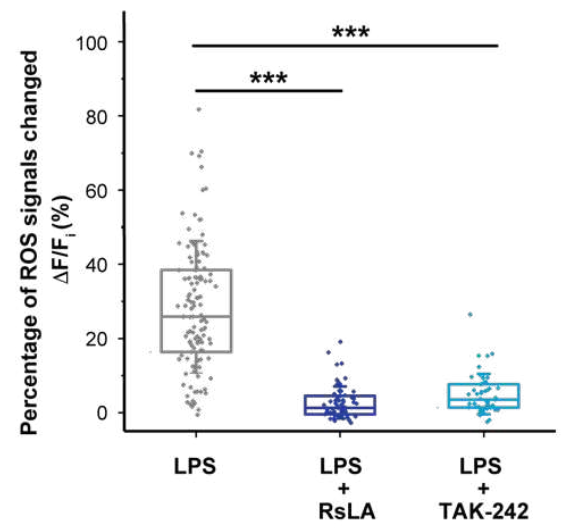

f

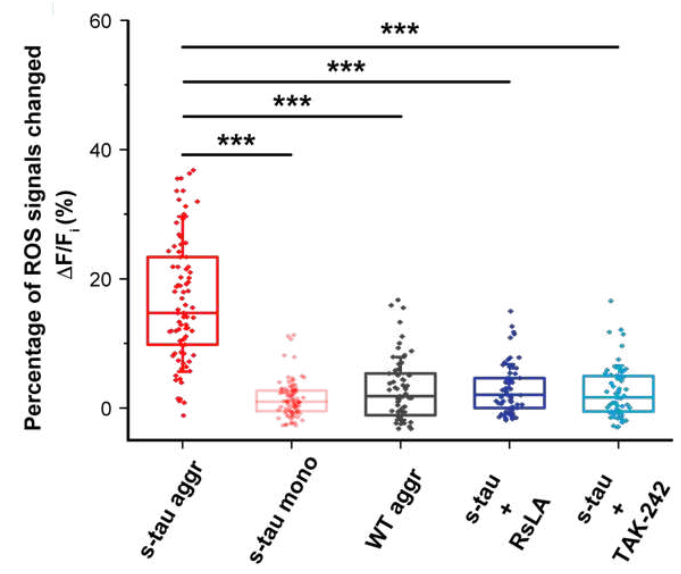


Figure 4. Hyperphosphorylated tau aggregates elicited calcium transient and stimulated ROS production in human macrophages in a TLR-4 dependent manner.

a. A pictorial representation of the macrophage assay workflow. The cytosolic calcium and ROS level were simultaneously monitored as different tau aggregates were applied.

b. Representative image of dual-labeled macrophage (i) and calcium and ROS traces (ii) after the treatment of s-tau aggregates. Scale bar: $25.0 \mu \mathrm{m}$.

c-d. Summary plots of calcium transients (c) and ROX production (d) induced by LPS ( $\mathrm{n}=$ 123). When treating the cells with RsLA $\left(n=70, \mathrm{~F}_{(1,4)}=40.78\right)$ or TAK-242 $(\mathrm{n}=47$, $\left.\mathrm{F}_{(1,4)}=84.62\right)$, the LPS-induced calcium transients and ROS level elevation were significantly moderated.

e-f. Summary plots of calcium transients (e) and ROX production (f) induced by different tau species and treatment conditions. Compared to WT tau aggregates $(n=63)$, s-tau aggregates induced strong calcium transients and $\operatorname{ROS}$ production $\left(\mathrm{n}=83, \mathrm{~F}_{(1,4)}=\right.$ 174.88). Since s-tau monomer control did not elicit significant response $\left(\mathrm{n}=89 \mathrm{~F}_{(1,4)}=\right.$ 110.14), ATP and LPS contamination were controlled. The involvement of TLR4 was tested: by pre-treating the cells with RsLA $\left(n=65, \mathrm{~F}_{(1,4)}=184.60\right)$ or TAK-242 $(\mathrm{n}=62$, $\left.\mathrm{F}_{(1,4)}=170.11\right)$, s-tau can no longer elicit significant response.

For c and d, the $P$ values are based on two-way mixed ANOVA: all $p<0.001$ except the one between LPS and RsLA which is $p<0.01$. For $\mathrm{d}$ and $\mathrm{f}$, the $P$ values are based on unpaired Student's t test: ${ }^{* *} p<0.001$. Error bars in $\mathrm{c}$ and e represent \pm s.d. of three independent experiments.

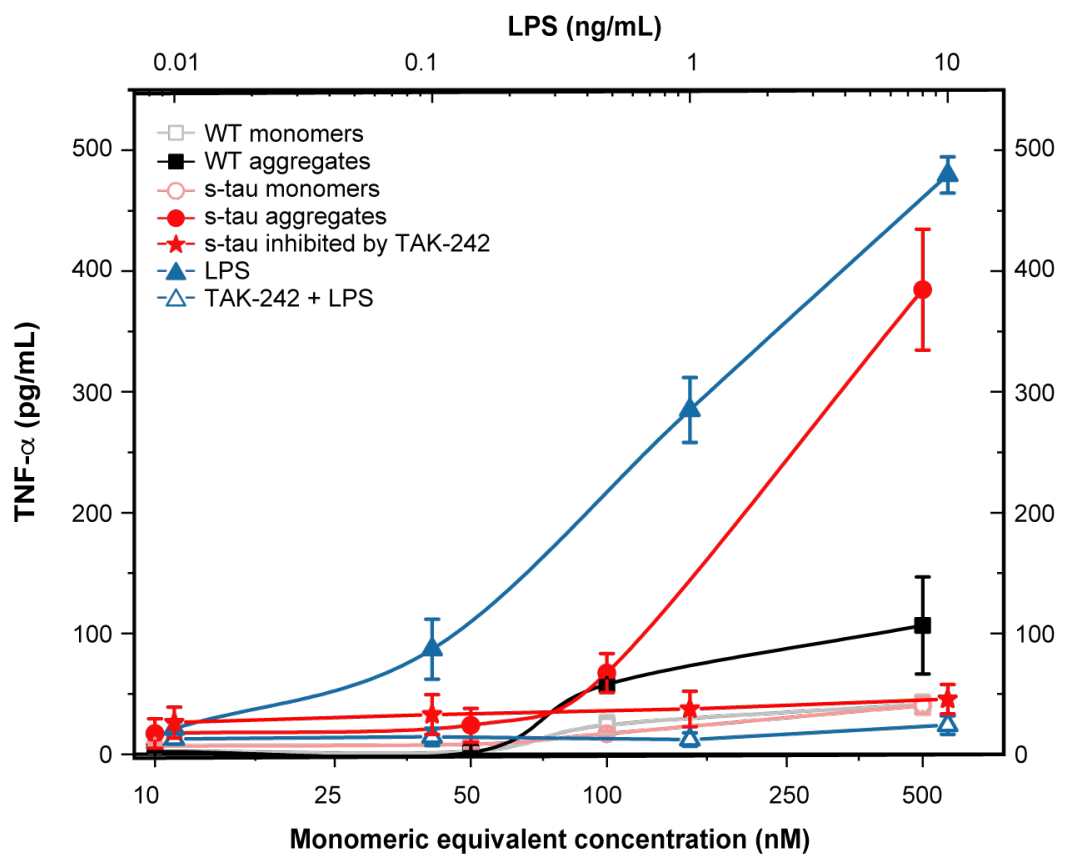


Figure. 5 Hyperphosphorylated tau elicited TLR4-mediated inflammation in human macrophages.

As a positive control, various concentrations of LPS (varying from $10 \mathrm{pg} / \mathrm{mL}$ to $10 \mathrm{ng} / \mathrm{mL}$ ) were applied to elicit robust TLR4 activation, resulting in the release of TNF- $\alpha$ in a dose-dependent fashion. Our results also showed s-tau aggregates can cause more potent proinflammatory response than WT aggregates after 24 hours of incubation $\left(F_{(1,7)}=67.07\right)$. Since monomer controls did not elicit any significant response in comparison with their corresponding aggregate samples $\left(\mathrm{F}_{(1,7)}=16.59\right.$ between WT aggregates and monomers, $\mathrm{F}_{(1,7)}=167.46$ between s-tau aggregates monomers), ATP and LPS contamination were controlled. The involvement of TLR4 was subsequently tested: the inhibitory effect of TAK242 was first being verified since the inhibitor successfully suppressed the level of TNF- $\alpha\left(\mathrm{F}_{(1,7)}=1120.74\right)$. When inhibited by TAK242 , the s-tau can no longer elicit any significant inflammatory response, thereby suggesting the observed inflammation caused by tau aggregated was mediated by TLR 4 . The $P$ values are based on one-way measures ANOVA: error bars represent \pm s.d. of $n=4$ independent experiments for each condition and all $p<0.001$. 
Phosphorylation sites specific to g-tau are highlighted in blue, Phosphorylation sites specific to s-tau are highlighted in red

Phosphorylation sites shared by both are highlighted in brown

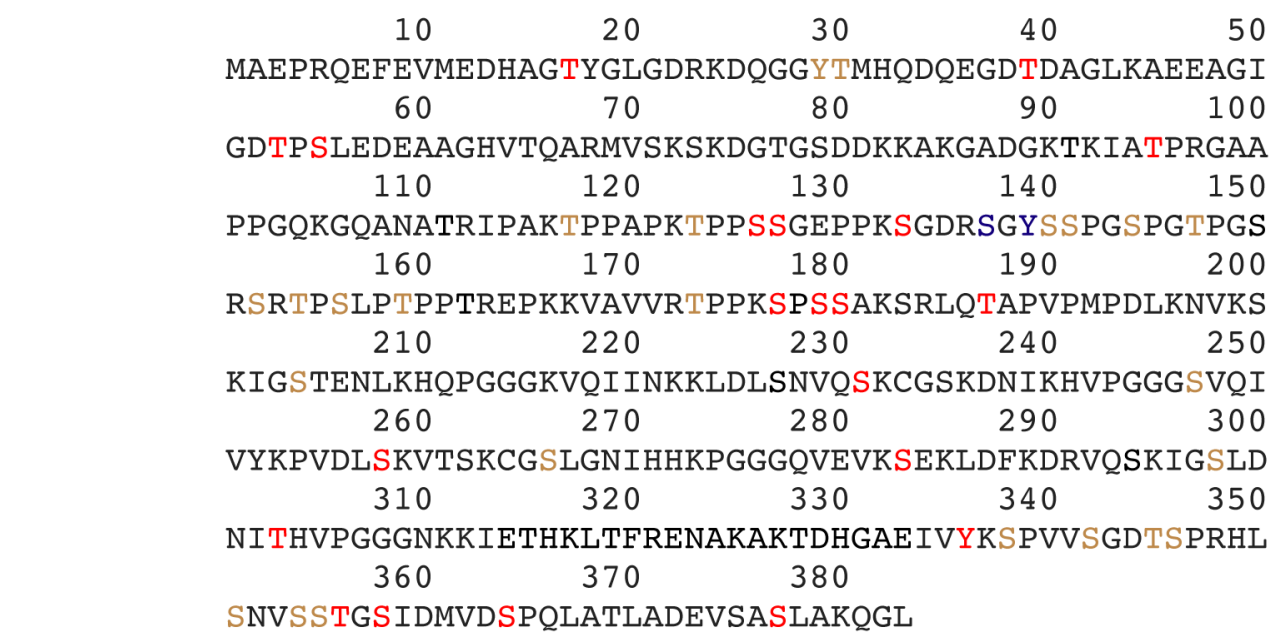

b
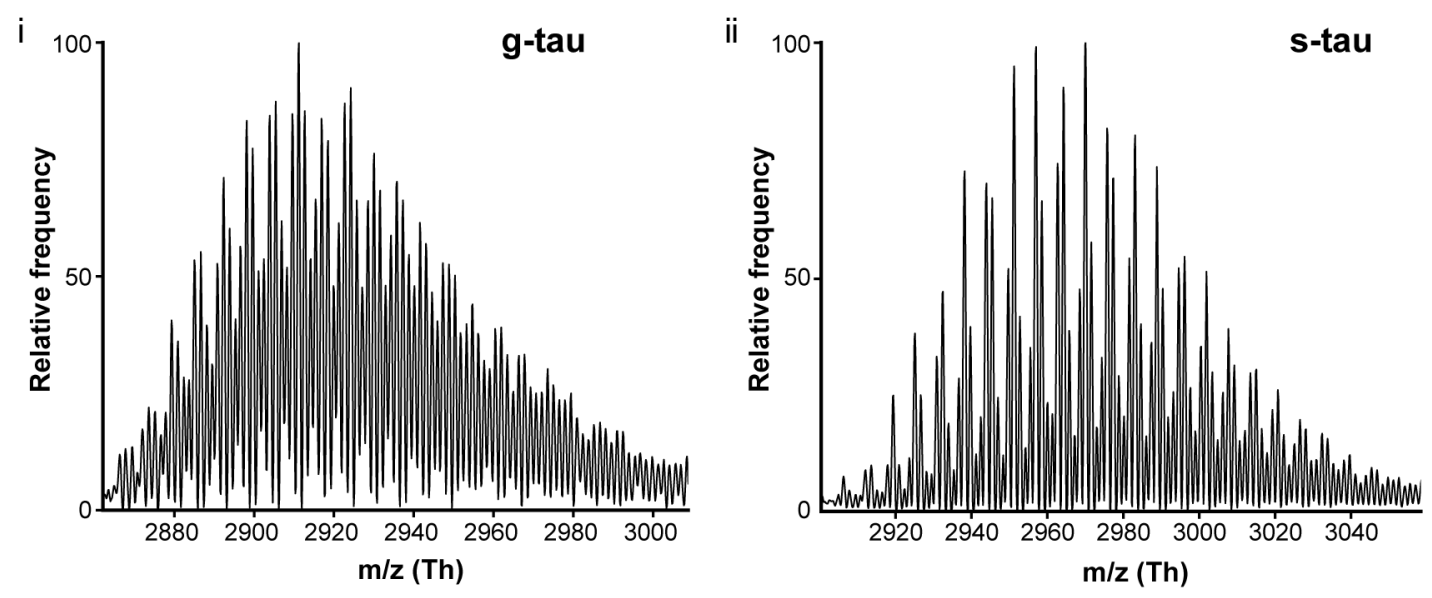

C
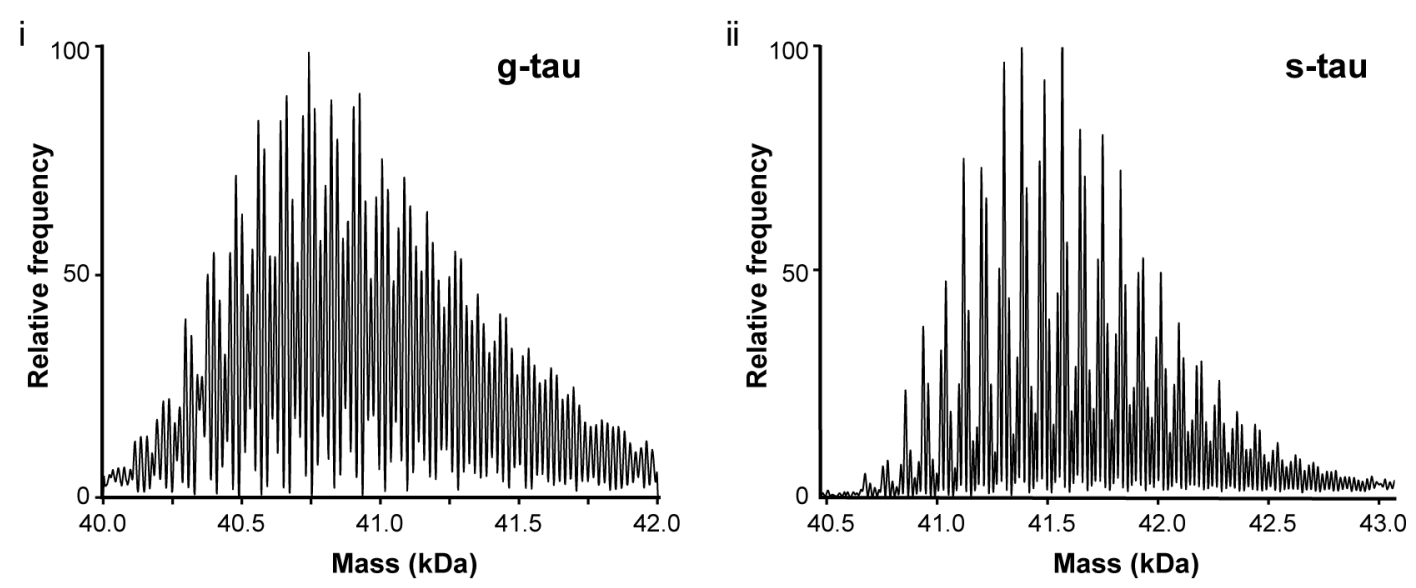


\section{SFigure 1. Characterization of the phosphorylation state of g-tau and s-tau by mass}

spectrometry.

a. LC-MS/MS analysis revealed that full-length tau was hyperphosphorylated with different specificities. Phosphorylation sites that were unique to GSK-3 $\beta$ are highlighted in blue, while the sites that were rather specific to SAPK4 are highlighted in red. Brown sites refer to the common sites phosphorylated by both kinases.

b. Representative raw data from high-resolution native mass spectrometry for g-tau and stau.

c. Representative deconvoluted mass spectrometry results indicated g-tau ranged between 40.0 and $42.0 \mathrm{kDa}$ which corresponded to 1-25 phosphate groups (80 Da each) per tau molecule. Meanwhile, s-tau exhibited a mass distribution between 40.5 and $43.0 \mathrm{kDa}$ which was equivalent to 1-32 phosphate groups per tau molecule.

a
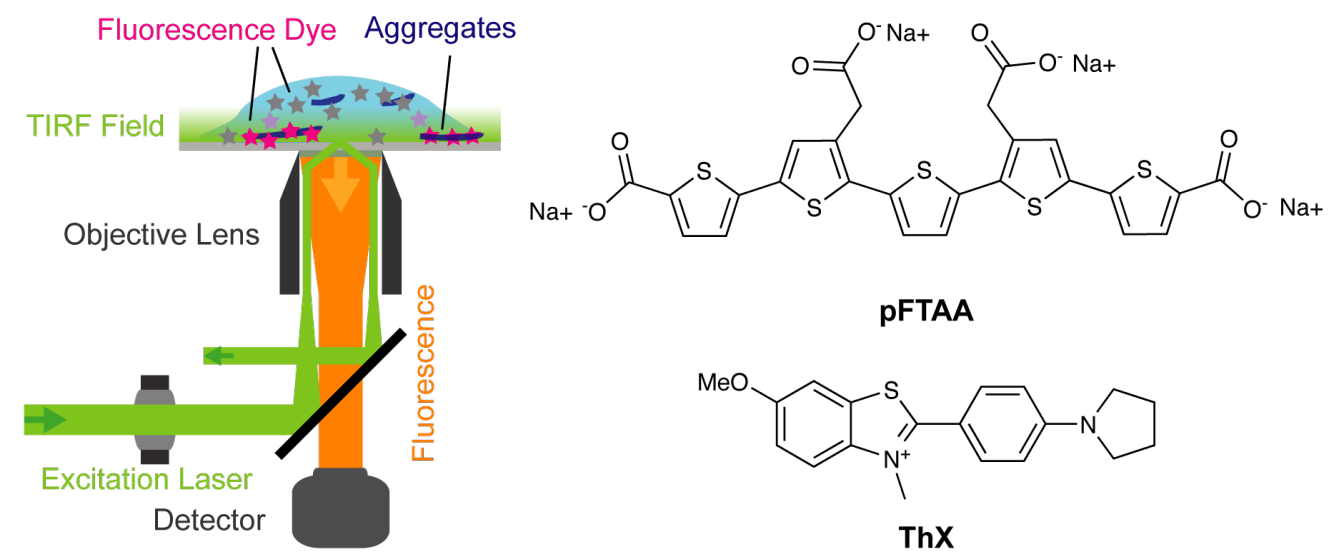

b
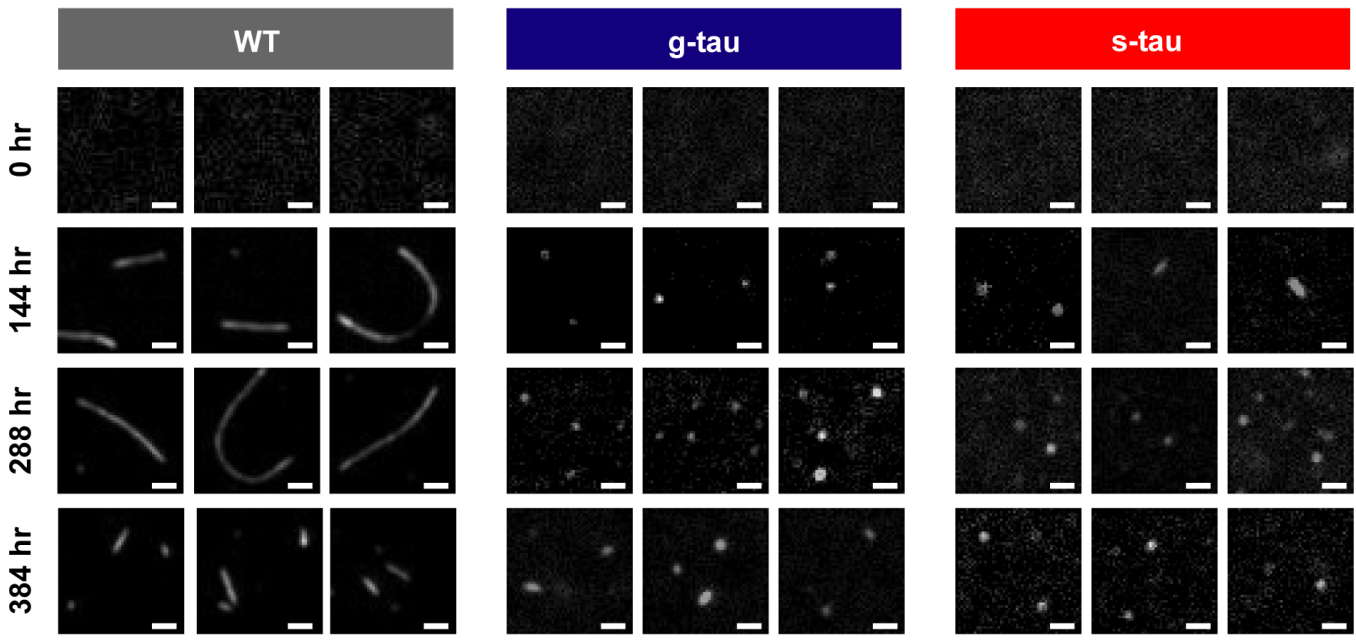
SFigure 2. Morphological characterization of aggregates formed by different tau species using fluorescence microscopy.

a. A pictorial representation of the TIRF microscopy setup workflow along with molecular structures of the fluorescence dyes being used, namely pFTAA and ThX.

b. Representative diffraction-limited images of different tau species at different timepoints of incubation: WT tau grew into fibrillar aggregates while both g-tau and s-tau formed small nonfibrillar aggregates. Scale bar: $1.0 \mu \mathrm{m}$.

a

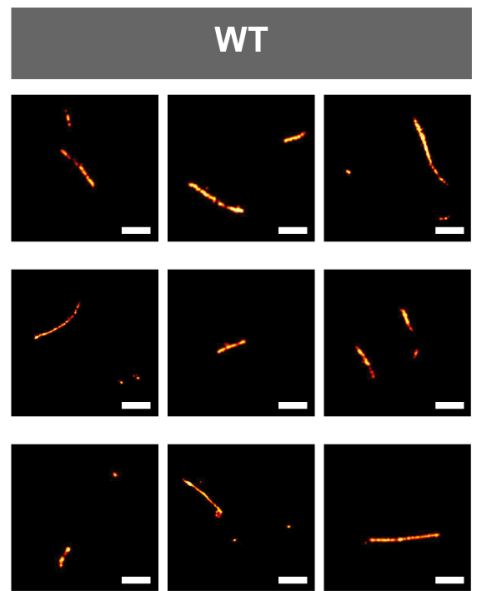

d

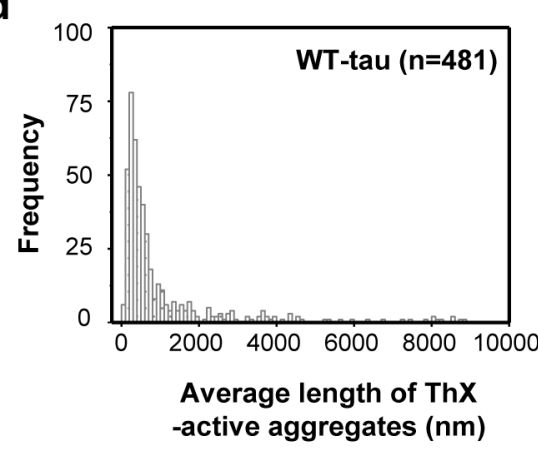

b
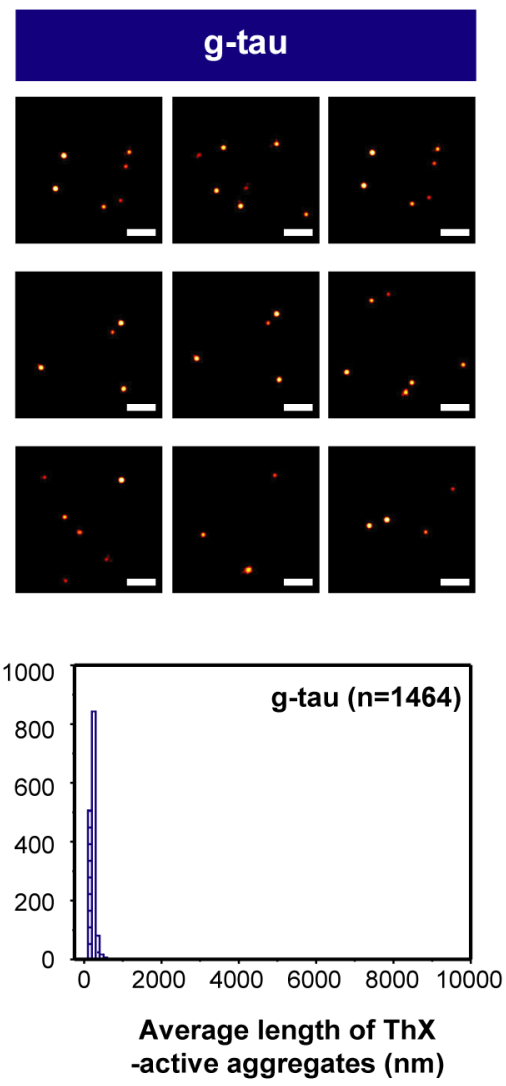

C
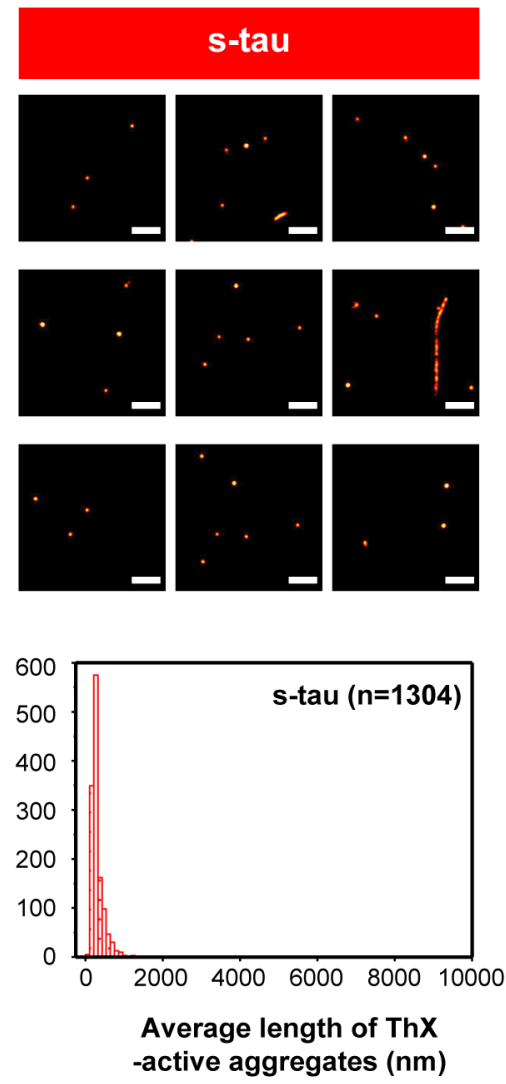

SFigure 3. Super-resolution imaging of tau aggregates.

a. Representative super-resolved images of WT aggregates after $96 \mathrm{hr}$ of incubation. Scale bar: $1.0 \mu \mathrm{m}$.

b. Representative super-resolved images of g-tau aggregates after $96 \mathrm{hr}$ of incubation. Scale bar: $1.0 \mu \mathrm{m}$.

c. Representative super-resolved images of s-tau aggregates after $96 \mathrm{hr}$ of incubation. Scale bar: $1.0 \mu \mathrm{m}$.

d. Size distribution histograms for different tau aggregates after $96 \mathrm{hr}$ of incubation. More than $25 \%$ of WT tau aggregated to fibrillar aggregates that were longer than $1000 \mathrm{~nm}$, 
whereas no g-tau and only $1.2 \%$ of s-tau were able to do so. On the other hand, about $99 \%$ of g-tau and $89 \%$ of s-tau aggregates stayed below a length of $500 \mathrm{~nm}$, while only half of WT tau aggregates were under that threshold.

a

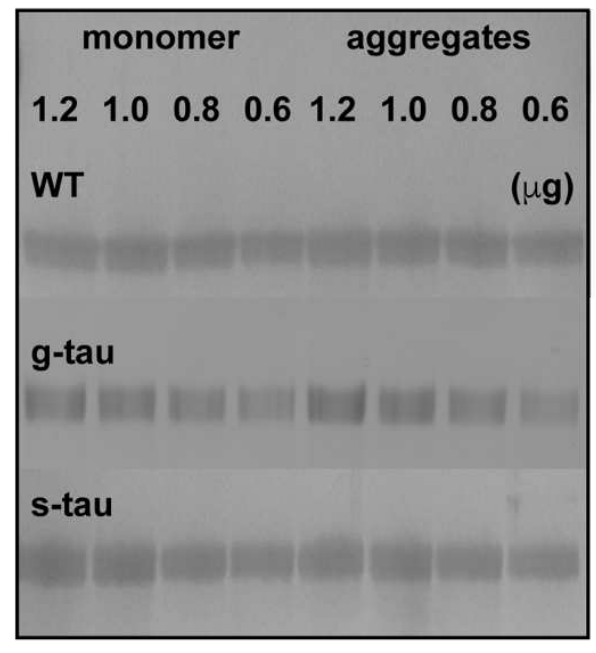

b

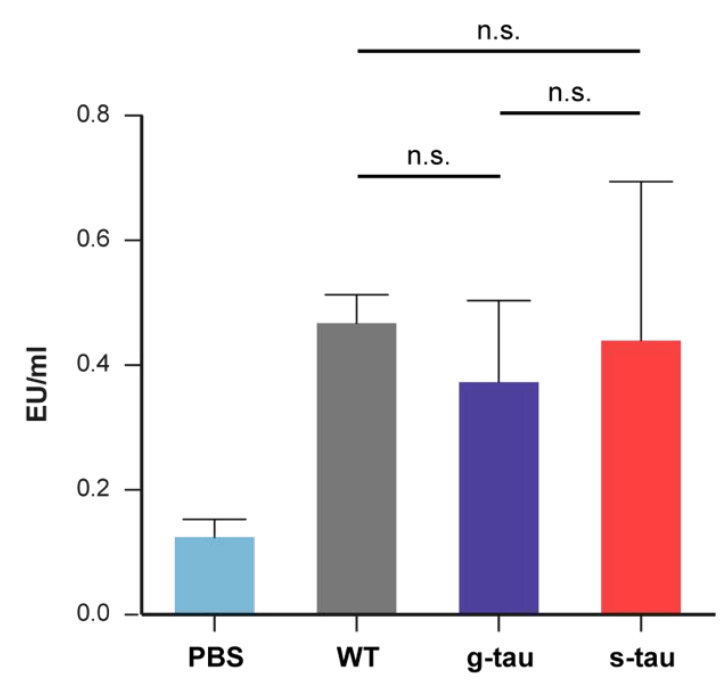

SFigure 4. Quantification of the degree of aggregation and residual LPS level for each tau species.

a. Representative SDS-PAGE results (10\% self-cast gel stained by Coomassie blue) quantified by densitometry allowed us to estimate the extent of aggregation at $96 \mathrm{hr}$ time point. By running various concentrations of tau aggregates and monomer from $0.6 \mu \mathrm{g}$ to $1.2 \mu \mathrm{g}$, we first confirmed the selected concentrations were within the linear range of our densitometric detection. Then by comparing the band intensities between aggregates and monomer, our SDS-PAGE result demonstrated that about $2 \%$ of WT tau and $1 \%$ of g-tau and s-tau had formed aggregates.

b. To ensure that our results were not confounded by endotoxins contamination of our recombinant tau proteins purified from bacterial cultures, LPS contamination was removed after the phosphorylation reactions by using Pierce ${ }^{\mathrm{TM}}$ high capacity endotoxin removal spin columns. To confirm the efficacy of this removal procedure, the LAL assay was employed to quantify the levels of any remaining LPS. At $2 \mu \mathrm{M}$ monomer equivalent concentration WT tau contained $0.47 \pm 0.05 \mathrm{EU} / \mathrm{mL}$, g-tau, $0.37 \pm 0.13 \mathrm{EU} / \mathrm{mL}$, and s-tau $0.43 \pm 0.25 \mathrm{EU} / \mathrm{mL}$ with PBS buffer control $0.13 \pm 0.03 \mathrm{EU} / \mathrm{mL}$, all of which are classified as low levels of LPS contamination $(0.5 \mathrm{EU} / \mathrm{mL})$ in cell cultures (Error bars represent \pm s.d. of $\mathrm{n}=3$ for each sample). The $P$ values are based on unpaired Student's t test: n.s., non-significant. 
a

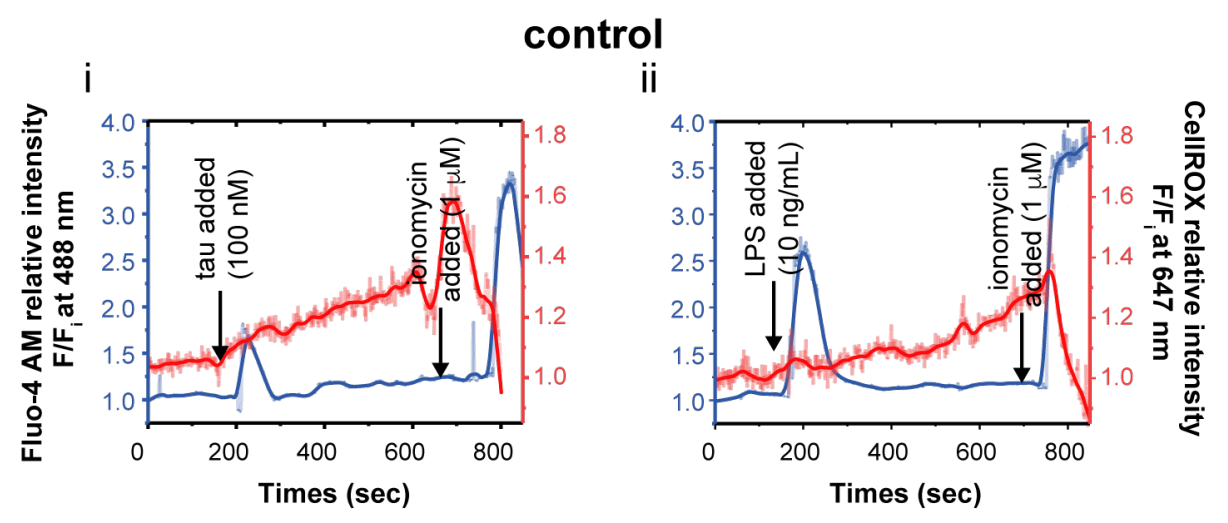

b

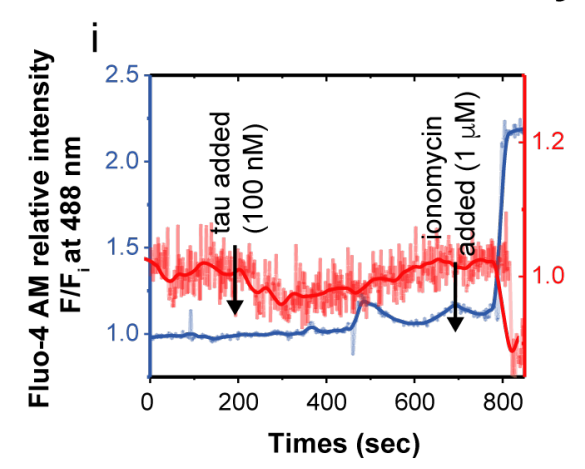

ii

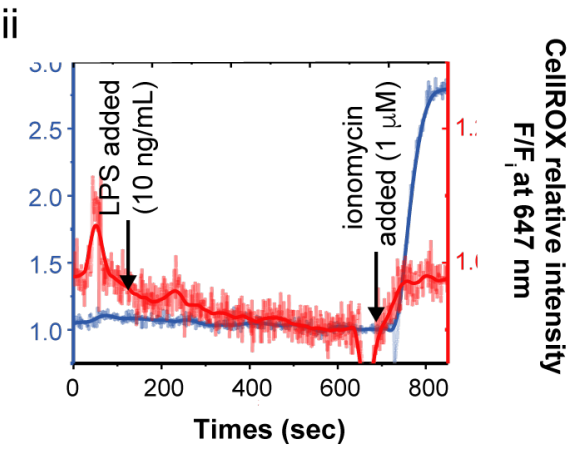

C

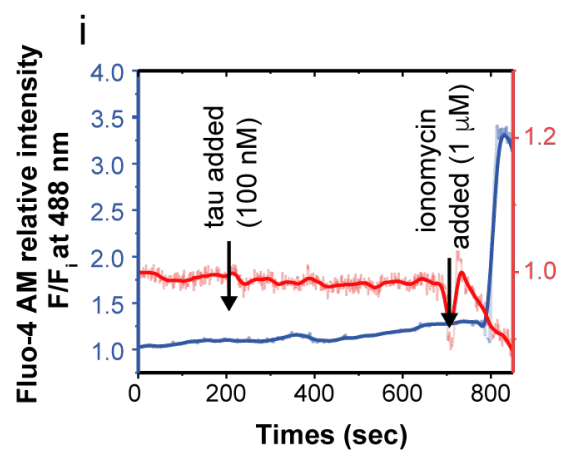

Inhibited by $5 \mu \mathrm{g} / \mathrm{mL}$ of RsLA

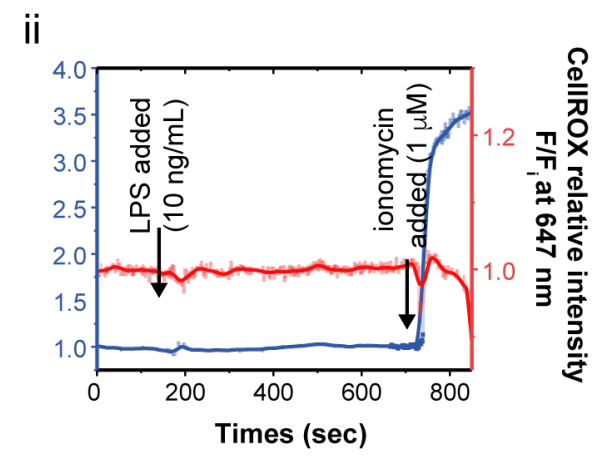

SFigure 5. TLR-4 inhibitors can effectively prevent calcium transients and ROS production elicited by s-tau aggregates in human macrophages.

Representative calcium and ROS signal traces after the application of corresponding stimuli, either s-tau aggregates at $100 \mathrm{nM}$ or LPS at $10 \mathrm{ng} / \mathrm{mL}$ under different conditions. In comparison with the control condition in a, TAK-242 in b and RsLA c exhibited strong inhibitory effect towards s-tau aggregates and LPS the canonical TLR4 agonist in terms of macrophage activation, suggesting s-tau stimulated calcium transient and ROS production through a TLR-4 dependent manner. 


\section{Methods}

\section{Protein expression and purification}

Human 0N4R tau was expressed as previously described ${ }^{20}$. In brief, pRK172 plasmids (gift from Michel Goedert) expressing full-length tau (isoform 0N4R) wild type were transformed in BL21(DE3) cells. Transformed E. coli BL21 cells were grown in Luria Broth media containing $100 \mu \mathrm{g} / \mathrm{mL}$ ampicillin at $37^{\circ} \mathrm{C}$ under shaking conditions until $\mathrm{OD}_{600}$ of 0.6 was reached, and tau expression was induced by addition of $1 \mathrm{mM}$ IPTG for 4 hours. Cells were then harvested by centrifugation at 4,000 g (JA-20 rotor, Beckman Coulter) for 30 minutes at $4{ }^{\circ} \mathrm{C}$, resuspended in lysis buffer (50 mM MES [pH 6.0] with 2,5 mM TCEP, $1 \mathrm{mM}$ AEBSF $)$, and lysed using a probe sonicator $(1 \mathrm{x} 1.5 \mathrm{~min}$, $5 \mathrm{~s}$ on, $10 \mathrm{~s}$ off, $40 \%$ amplitude). The cell debris was subsequently removed by centrifugation for 30 minutes at $18,000 \mathrm{~g}$ at $4{ }^{\circ} \mathrm{C}$. RNase and DNase were added before the supernatant was filtered and loaded onto a Resource S ion exchange column (GE Healthcare). Protein was eluted over a linear $\mathrm{NaCl}$ gradient from 0 to $500 \mathrm{mM}$, and fractions containing tau protein (determined by SDS-PAGE) were pooled and precipitated with $20 \%$ ammonium sulfate at $4{ }^{\circ} \mathrm{C}$ for 1 hour. The protein was pelleted at 15,000 g for 20 minutes at $4{ }^{\circ} \mathrm{C}$. After the pellet was resuspended in SSPE buffer with $2.5 \mathrm{mM}$ TECP and $0.1 \mathrm{mM}$ PMSF, the protein was further purified using size-exclusion chromatography with a Superdex 200 Increase 10/300 size exclusion column (GE Healthcare Life Sciences). The fractions were collected analyzed by SDS-PAGE, and those having the purest bands corresponding to tau were pooled. The protein concentration was then determined by measuring its absorbance at $280 \mathrm{~nm}$ on a Nanodrop $2000 \mathrm{using}$ an extinction coefficient of 7450 $\mathrm{M}^{-1} \mathrm{~cm}^{-1}$ and by BCA assay (Thermo Fisher) following the manufacture protocol. Aliquots were flash-frozen in liquid nitrogen and stored at $-80{ }^{\circ} \mathrm{C}$.

\section{In vitro phosphorylation of recombinant tau}

Full-length recombinant tau (0N4R) was sequentially phosphorylated as described previously ${ }^{13}$. Monomeric tau was incubated at a final concentration of $50 \mu \mathrm{M}$ in 25 
$\mathrm{mM}$ Tris-HCl buffer [pH 7.4] (Sigma-Aldrich) with $0.1 \mathrm{mM}$ ethylene glycol tetraacetic acid (EGTA, Sigma-Aldrich), 2 mM AEBSF protease inhibitor (SigmaAldrich), $10 \mathrm{mM}$ magnesium acetate, $2 \mathrm{mM} \mathrm{ATP}$ (Sigma-Aldrich). $0.018 \mathrm{U}$ of the catalytic subunits of cAMP-dependent protein kinase A (PKA, BioVision) per nmol of tau was added and the reaction was incubated at $30{ }^{\circ} \mathrm{C}$. After 24 hours, the second kinase (GSK-3 $\beta$ or SAPK4 from Abcam, $0.018 \mathrm{U}$ per nmol of tau) was added and the reaction proceeded for an additional 24 hours. One unit of the kinase is defined as the amount of enzyme that will transfer one nmol phosphate from ATP to the corresponding substrate per min at $\mathrm{pH} 7.4$ at $30^{\circ} \mathrm{C}$, tested and reported by individual manufacturers. Phosphorylation was confirmed sodium dodecyl sulfate polyacrylamide gel electrophoresis (SDS-PAGE). Residual LPS is also known as endotoxin derived from the outer cell membrane of gram-negative bacteria can elicit TLR4 signaling. To ensure that our results were not confounded by the residual LPS in our tau proteins purified from bacterial cultures, LPS contamination were effectively eliminated after phosphorylation reactions by using high capacity endotoxin removal spin columns (Thermo Fisher). This column has been shown to effectively remove contaminating endotoxins (i.e. residual lipopolysaccharides) in protein samples purified from bacterial cultures without introducing other bioactive species. Our endotoxin quantification results subsequently indicated that at $2 \mu \mathrm{M}$ WT tau contained $0.47 \pm 0.05 \mathrm{EU} / \mathrm{mL}$, g-tau, $0.37 \pm 0.13 \mathrm{EU} / \mathrm{mL}$, and s-tau $0.43 \pm$ $0.25 \mathrm{EU} / \mathrm{mL}$ with PBS buffer control $0.13 \pm 0.03 \mathrm{EU} / \mathrm{mL}$. After endotoxin removal, the protein concentration was then determined by Nanodrop and by BCA assay as mentioned above. Aliquots at $40 \mu \mathrm{M}$ were flash-frozen in liquid nitrogen and stored at $-80{ }^{\circ} \mathrm{C}$.

\section{Liquid chromatography-tandem mass spectrometry (LC-MS/MS)}

Detailed phosphorylation mapping was obtained from LC-MS/MS analysis conducted by Cambridge Center for Proteomics, and the measurements were repeated across three independent experiments for both $\mathrm{g}$-tau and s- tau proteins to ensure reproducibility. Phosphorylation sites observed across three repeats were highlighted in SFig. 1a for 
each species. Variations due to the behavior of the tryptic peptides in the instrument were minimal.

Protein solutions were reduced (DTT), alkylated (iodoacetamide) and enzymatically digested in $50 \mathrm{mM}$ ammonium bicarbonate [pH 8] with trypsin overnight at $37^{\circ} \mathrm{C}$. After digestion, the supernatant was pipetted into a sample vial and loaded onto an autosampler for automated LCMS/MS analysis.

All LC-MS/MS experiments were performed using a Dionex Ultimate 3000 RSLC nanoUPLC (Thermo Fisher Scientific Inc, Waltham, MA, USA) system and a QExactive Orbitrap mass spectrometer (Thermo Fisher Scientific Inc, Waltham, MA, USA). Separation of peptides was performed by reverse-phase chromatography at a flow rate of $300 \mathrm{~nL} / \mathrm{min}$ and a Thermo Scientific reverse-phase nano Easy-spray column (Thermo Scientific PepMap C18, $2 \mu \mathrm{m}$ particle size, $100 \mathrm{~A}$ pore size, $75 \mu \mathrm{m}$ i.d. x 50cm length). Peptides were loaded onto a pre-column (Thermo Scientific PepMap 100 C18, $5 \mu \mathrm{m}$ particle size, 100A pore size, $300 \mu \mathrm{m}$ i.d. x 5mm length) from the Ultimate 3000 autosampler with $0.1 \%$ formic acid for 3 minutes at a flow rate of $10 \mu \mathrm{L} / \mathrm{min}$. After this period, the column valve was switched to allow elution of peptides from the pre-column onto the analytical column. Solvent A was water $+0.1 \%$ formic acid and solvent $\mathrm{B}$ was $80 \%$ acetonitrile, $20 \%$ water $+0.1 \%$ formic acid. The linear gradient employed was 2 $40 \% \mathrm{~B}$ in 30 minutes.

The LC eluant was sprayed into the mass spectrometer by means of an Easy-Spray source (Thermo Fisher Scientific Inc.). All m/z values of eluting ions were measured in an Orbitrap mass analyzer, set at a resolution of 35000 and was scanned between $\mathrm{m} / \mathrm{z} 380-1500$. Data dependent scans (Top 20) were employed to automatically isolate and generate fragment ions by higher energy collisional dissociation (HCD, NCE:25\%) in the HCD collision cell and measurement of the resulting fragment ions was performed in the orbitrap analyzer, set at a resolution of 17500. Singly charged ions and ions with unassigned charge states were excluded from being selected for MS/MS and a dynamic exclusion window of 20 seconds was employed. 
Post-run, all MS/MS data were converted to mgf files and the files were then submitted to the Mascot search algorithm (Matrix Science, London UK) and searched against the UniProt Human database (93609 sequences; 37041084 residues) and a common contaminant sequences containing non-specific proteins such as keratins and trypsin (125 sequences; 41129 residues). Variable modifications of oxidation (M), deamidation (NQ) and phosphorylation (S,T,Y) were applied as well as fixed modification of carbamidomethyl (C). The peptide and fragment mass tolerances were set to $20 \mathrm{ppm}$ and $0.1 \mathrm{Da}$. A significance threshold value of $\mathrm{p}<0.05$ and a peptide cut-off score of 20 were also applied.

\section{High-resolution native mass spectrometry}

The protein samples were stored in phosphorylation reaction buffer at $-80{ }^{\circ} \mathrm{C}$ before being buffer exchanged into $200 \mathrm{mM}$ ammonium acetate [pH 6.8] by multiple rounds of concentration and dilution using the Pierce ${ }^{\mathrm{TM}}$ protein concentrators (Thermo Fisher). Finally, the samples were diluted to monomer equivalent concentration of $1 \mathrm{mM}$ before the measurements. The data was collected using in-house gold-plated capillaries on a Q Exactive ${ }^{\mathrm{TM}}$ mass spectrometer in positive ion mode with source temperature of $70{ }^{\circ} \mathrm{C}$ and capillary voltage of $1.2 \mathrm{kV}$. In-source trapping was set to $-120 \mathrm{~V}$ to help with dissociation small ion adducts. Ion transfer optics and voltage gradient throughout the instruments were optimized for ideal transmission. Spectra were acquired with 10 microscans to increase the signal-to-noise ratio with transient times of $64 \mathrm{~ms}$, corresponding to resolution of 17,500 at $\mathrm{m} / \mathrm{z}=200$, and AGC target of $1.0 \times 10^{6}$. The noise threshold parameter was set to 3 and the scan ranges used were 346-14,147 and 1,000-10,000 $\mathrm{m} / \mathrm{z}$ for $\mathrm{g}$ - and s-tau respectively.

The measurements were repeated across three independent experiments for both g-tau and s- tau proteins to ensure reproducibility. Mass deconvolution was achieved using the UniDec software as previously described ${ }^{61}$. Before deconvolution, the data was pre-processed by setting the curved subtraction to ten, which resulted in significantly more baseline-resolved peaks. Further, the fitted peak FWHM was optimized for each spectrum (generally within 0.5 and $1 \mathrm{Th}$ ). All other UniDec parameters were left at their default values. The deconvolution was performed in the mass range of $40-44 \mathrm{kDa}$. 
The estimation of the degree of phosphorylation was complicated by small ion adducts present in the deconvoluted mass spectra, which extend the range of masses associated with any particular phosphorylation state, as well as possibly cause some mass overlap. To find the optimal distribution of phosphate groups that explained the deconvoluted mass spectrum, as well as the uncertainty of this assignment, a Markov Chain Monte Carlo assignment method was employed. This was done using the emcee python package as previously described ${ }^{62}$ ([1202.3665] emcee:

The MCMC Hammer (arxiv.org)). The masses of one phosphate group and one small ion adduct were set to 80 and $23 \mathrm{Da}$, respectively. The mass of the small ion adduct was chosen so that it is within the integration window of $10 \mathrm{Da}$. Two most probable adducts were the ammonium and sodium cations. The priors used for fitting were that the number of the phosphate groups and small ion adducts which were kept below 50, independently. The limits were chosen for time efficiency and to keep make sure the combined mass of the phosphate groups was not likely to go above the $44 \mathrm{kDa}$ limit of the deconvolved mass spectrum. 6 chains were run for 3,000 steps each: the first 800 steps were discarded as a burn-in, and the rest was thinned by a factor of 100 to reduce any auto-correlation. The thinned chains were then used to estimate the extent of phosphorylation by summing the area under the curve (to account for the inherent broadness of the deconvolved mass peaks, as well as the fact that there are two possible small adduct ions) in corresponding mass regions using a mass window of $10 \mathrm{Da}$.

\section{Standard tau aggregation procedure}

Unphosphorylated and phosphorylated tau protein was used. Monomers were incubated at $2 \mu \mathrm{M}$ in PBS buffer $\left(137 \mathrm{mM} \mathrm{NaCl}, 3 \mathrm{mM} \mathrm{KCl}, 8 \mathrm{mM} \mathrm{Na}_{2} \mathrm{HPO}_{4}, 1.5\right.$ $\mathrm{mM} \mathrm{KH}_{2} \mathrm{PO}_{4}, \mathrm{pH} 7.3$ ) with $0.01 \% \mathrm{NaN}_{3}$ at $37{ }^{\circ} \mathrm{C}$. Heparin (Fisher Scientific)was added at $10 \mu \mathrm{g} / \mathrm{mL}$ (about $2 \mu \mathrm{M}$ ) to induce aggregation. Self-assembly reactions were rather carried out in the absence of heparin.

\section{SDS-PAGE}

Protein samples along with molecular weight marker (SeeBlue ${ }^{\text {TM }}$ Plus 2 Pre-Stained Standard) were load into the wells of 4\%-12\% gradient NuPAGE Bis-Tris precast 
gels or $10 \%$ self-cast SDS-PAGE gels and run in MOPS-based SDS running buffer for 55 minutes at $200 \mathrm{~V}$. The proteins were visualized by Coomassie stain.

\section{Single-molecule TIRF Microscopy}

\section{Instrumentation}

The samples were imaged using a home-built total internal reflection fluorescence (TIRF) microscope. The total internal reflection mode limits the fluorescence field to $200 \mathrm{~nm}$ from the sample slide. For pFTAA imaging, a $488 \mathrm{~nm}$ diode laser (TOPTICA iBeam smart, $175 \mathrm{~mW}$ ) was aligned and directed to the optical axis at the edge of a TIRF objective (Apo TIRF 100x/NA1.49 oil objective, Nikon) mounted on an inverted microscope (Eclipse Ti-S, Nikon). The emitted fluorescence was collected by the same objective, separated from the returning TIRF beam by a dichroic mirror (Di01-R405/488/532/635-25x36, Semrock), and passed through an emission filter (FF552-Di02-25x36, Semrock). For ThX super-res imaging, the same $488 \mathrm{~nm}$ laser was used, but a different set of emission filters (LP02-568RS-25, FF01-587/35-25, Semrock) was utilized to acquire the optimal super-resolution images. The control of the hardware was enabled by custom-written scripts for MicroManager (NIH). The signal was recorded on an Evolve Delta 512 EMCCD camera (Photometrics) Each pixel was $107.2 \mathrm{~nm}$ in length such that the dimensions of a single image taken were 54.8 by $54.8 \mu \mathrm{m}^{2}$.

The switch from TIRF to widefield mode was accomplished by altering the beam offset perpendicular to the optical axis. To simultaneously track calcium transients and ROS level changes, a $488 \mathrm{~nm}$ diode laser (TOPTICA iBeam smart, $175 \mathrm{~mW}$ ) and a $638 \mathrm{~nm}$ laser (Cobolt 06-MLD, $180 \mathrm{~mW}$ ) were aligned and directed to the optical axis of a 20x objective (Nikon) mounted on an inverted microscope (Eclipse T $i$-S, Nikon). The emitted fluorescence was collected by the same objective, separated from the returning beam by a dichroic mirror (Di01-R405/488/532/635$25 \times 36$, Semrock), and passed through an emission filter (FF580-FDi01-25x36, Semrock for $488 \mathrm{~nm}$ excitation and BLP01-635R-25, Semrock for $638 \mathrm{~nm}$ 
excitation). Each channel was recorded alternatingly at a 1 second temporal resolution, and each frame had an exposure time of $100 \mathrm{~ms}$.

\section{Sample preparation for TIRF imaging}

Prior to imaging, Borosilicate glass coverslips (Ø50 mm, VWR International) were cleaned using an argon plasma cleaner (PDC-002, Harrick Plasma) for 1 hour to remove any autofluorescent residue. Multiwell slide chambers (CultureWell chambered cover glass 50 well, Grace Bio-Laboratories) were affixed onto the cleaned coverslips. To stain the aggregates for diffraction-limited imaging, samples were diluted in PBS buffer containing $30 \mathrm{nM}$ pFTAA (gift from Michel Goedert) to a final monomer equivalent concentration of $67 \mathrm{nM}$ tau. Then $15 \mu \mathrm{L}$ of each sample was applied to each well on the coverslip for 15 minutes before imaging. Images were recorded for 100 frames, each with an exposure time of $50 \mathrm{~ms}$. The image stacks were averaged using ImageJ (NIH) software for further analysis.

To super-resolve the structures of tau aggregates, especially the early aggregate species whose lengths are under $100-200 \mathrm{~nm}$, ThX, a synthetic variant of welldocumented Thioflavin T (ThT), was used. After the coverslips being plasma cleaned, the surface was washed with PBS buffer and coated with poly-L-lysine (PLL, $1 \mathrm{mg} / \mathrm{mL}$ ) for 15 minutes to reduce non-specific binding events. After gently removing the excess PLL coating, gold nanoparticles with $200 \mathrm{~nm}$ diameter size were applied onto the surface for 5 minutes, serving as fiducial markers to correct thermal drift during imaging. The surface was then incubated with protein aggregates for 20 minutes. Subsequently, samples were imaged in the presence of 1 $\mu \mathrm{M}$ ThX dye dissolved in PBS buffer. Note that one or two washes with PBS between each surface treatment procedure, i.e. PLL coating, gold nanoparticles application, and protein incubation, are recommended to obtain the optimal imaging condition. Images were recorded for 10,000 frames, each with an exposure time of $20 \mathrm{~ms}$. 


\section{Diffraction-limited image analysis}

For each sample, 16 fields of view were typically recorded. Individual image data were averaged over all the framed by ImageJ (NIH) software and then analyzed with a custom-written MATLAB script as previously described ${ }^{63}$ (R2018b, MathWorks) to count the total number of fibrils and aggregates, the size, and fluorescence intensity. Since both the concentrations of protein and pFTAA were controlled in each experiment, the number and the intensity of fibrils and aggregates can be directly compared across different experiments. For particle identification, images were bandpass filtered to remove the modulated background and camera noise. To identify particle boundaries, the foreground was then blurred using a 2D Gaussian filter with a threshold applied based on the pixel intensity with a criterion of $2 \%$ intensity above the background (median value of the whole image). The length of aggregates was measured by thinning boundaries of individual particles and then calculated with an image pixel size of $107.2 \mathrm{~nm}$ for our TIRF setup. To eliminate the background effect in our intensity calculation, single-to-background ration (SBR) was introduced to correct the intensity of pixels, where the SBR is defined as:

$$
S B R=\frac{\text { intensity above the background }}{\text { Background }}
$$

For a given particle, its corrected intensity is the sum of each pixel's SBR values within its boundary.

\section{Super-resolution Image Analysis}

The raw image stacks were passed through a custom-written Python scriptas previously described ${ }^{64}$. We defined a localization as $2 \mathrm{D}-$ Gaussian fit of a fluorescence signal in one image frame. The localizations were determined using the 'Peak Fit' plugin within Fiji (ImageJ)/GDSC Single Molecule Light Microscopy package

(http://www.sussex.ac.uk/gdsc/intranet/microscopy/UserSupport/AnalysisProtocol/i magej/gdsc_plugins/). The adjustable parameters 'signal strength' and 'precision' 
were set to 3 and $30 \mathrm{~nm}$ respectively. These values referred to the properties of the 2D Gaussian fit and were set by visual inspection of the rendered localization images. The fiducial markers of the images were pinpointed as localizations that lasted more than 500 frames at the same location. Fiducial signals were first removed from localization file from the subsequent analysis.

Then image stacks went through temporal grouping, which sorted localizations into bursts. Temporal grouping was achieved by: 1) using DBSCAN clustering function (scikit-learn) on the spatial domain (XY coordinates) of the localizations, with a detection radius (epsilon) of $15 \mathrm{~nm}$ and minimum point threshold of 3. 2) using DBSCAN again on the temporal domain (frame number) with an epsilon of $21 \mathrm{~ms}$ and a minimal frame number of 2 to recognize individual bursts and remove single frame localizations. The rationale of the settings here is that many non-specific binding events are characterized by short transient signals that last less than the exposure time of the camera. Finally, the burst information was processed through a final DBSCAN cluster analysis on the spatial domain using an epsilon of $200 \mathrm{~nm}$ and a minimal burst number of 20 to group the bursts into clusters, which were interpreted as detections of individual super-resolved protein aggregates.

For each cluster analyzed, the number of bursts ranged from 34 to 3,356 . Subsequently, we structurally characterized the sizes of clusters by 1) scaling the spatial domain by 8 times and rounding the coordinates to integers; 2) closing the morphological space between bursts using the scikit-image 'closing' function; 3 ) skeletonizing the closed shape to a width of a single pixel using the scikit-image 'skeletonize_3d' function; 4) calculating the lengths by traversing the skeleton and recording the 8 -connectivity distances. Finally, the clusters were loaded into the 'Results Manager' plug-in in Fiji/GDSC SMLM and rendered into super-resolution images with each cluster labelled and characterized. 


\section{In Vitro Membrane Permeabilization Assay}

Liposomal penetration assay was conducted as described previously ${ }^{24}$. To prepare the lipid vesicles, 16:0-18:1 PC (10 mg/mL) and 18:1-12:0 Biotin PC $(1 \mathrm{mg} / \mathrm{mL})$ (Avanti Lipids) were mixed with 100:1 ratio. The lipid mixture was then hydrated in HEPES buffer (50 mM, pH 6.5) with $100 \mu \mathrm{M}$ Cal-520 (Stratech). Five freeze-andthaw cycles were performed using a water bath and dry ice to acquire the unilamellarity. The lipid solution was extruded at least 10 times through a membrane with a size cut-off of $200 \mathrm{~nm}$, and the size of the vesicles was measure using a Zetasizer (Zetasizer Nano ZSP). Free dyes were removed using size exclusion chromatography.

To prepare the surface, glass coverslips (VWR International, product number 63 10122 ) were cleaned by sonicating in 2\% (v/v) Hellmanex III (Hellma GmbH \& Co. KG) in Milli-Q water for 10 minutes followed by sonicating twice in Milli-Q water and methanol for 10 minutes, respectively. Then the coverslips were dried under a stream of nitrogen gas, and plasma-etched using an argon plasma cleaner (PDC-002, Harrick Plasma) for 1 hour to remove any fluorescent impurities. Each cover slide was fixed by frame-seal incubation chambers (Biorad, Hercules) and the surface was coated with 100:1 PLL-g-PEG and PLL-g-PEG biotin (SuSoS AG) (1 g/L) in $50 \mathrm{mM}$ HEPES buffer. Then the coverslips were washed three times and $0.1 \mathrm{mg} / \mathrm{mL}$ neutravidin (Thermo Scientific) solution was added to the coverslips and incubated for $15 \mathrm{~min}$ and washed three times with reaction buffer. Then, a $50 \mu \mathrm{L}$ aliquot of the solution of purified biotinylated vesicles was added to the surface and incubated for 30 minutes before washing carefully at least five times with reaction buffer. Single vesicles tethered to borosilicate glass cover slides via biotin neutravidin linkage were incubated with $50 \mu \mathrm{L} \mathrm{Ca}^{2+}$ containing buffer solution Leibovitz's L-15 (phenol red-free) and background image was recorded ( $\left.F_{\text {background }}\right)$. Fifty microliter of sample was added and images were acquired $\left(\mathrm{F}_{\text {sample }}\right)$ and care was taken to avoid moving the glass coverslips during the addition of each sample. Next, $10 \mu \mathrm{L}$ of a solution containing $1 \mathrm{mg} / \mathrm{mL}$ of ionomycin, an ionophore for $\mathrm{Ca}^{2+}$ ion, and subsequently images of $\mathrm{Ca}^{2+}$ saturated single vesicles in the same fields of view 
were acquired ( $\left.F_{\text {ionomycin }}\right)$. For each field of view, 50 images were taken with an exposure time of $50 \mathrm{~ms}$. The fields of view were chosen using an automated program via ImageJ Micromanager. The relative influx of $\mathrm{Ca}^{2+}$ into an individual vesicle due to aggregates of tau was then determined as

$$
\text { Percent of } \mathrm{Ca}^{2+} \text { influx }=\frac{F_{\text {sample }}-F_{\text {background }}}{F_{\text {ionomycin }}-F_{\text {background }}}
$$

The average calcium influx was calculated by averaging the $\mathrm{Ca}^{2+}$ influx into individual vesicles in each field of view.

\section{TEM}

To prepare samples for TEM experiments, protein solutions at an appropriate concentration (typically $\sim 200 \mathrm{nM}$ ) were applied onto a carbon-coated 400-mesh copper grid (Agar Scientific) for 1 minute, and then stained with $2 \%(\mathrm{v} / \mathrm{v})$ uranyl acetate for another minute. The excess solution was removed from the grid by washing twice with Milli-Q water. After samples dried thoroughly, TEM images were acquired using Thermo Scientific (FEI) Talos F200X G2 microscope (Department of Chemistry, Cambridge) operated at $200 \mathrm{kV}$.

\section{Cell Culture}

Human monocytic THP-1 cells (ATCC) were cultured in Roswell Park Memorial Institute medium (RPMI 1640, Invitrogen) culture medium supplemented with 10\% of heat-inactivated fetal bovine serum (Invitrogen) in $5 \% \mathrm{CO}_{2}$ humidified atmosphere at $37^{\circ} \mathrm{C}$. THP- 1 monocytes were first seeded at 200,000 cell $/ \mathrm{mL}$ on sterilized glass coverslips. Then THP-1 cells were differentiated into macrophages in the presence of $20 \mathrm{ng} / \mathrm{mL}$ phorbol 12-myristate 13-acetate (PMA, Sigma-Aldrich) for 3 trials continuously. This was followed a recovery period of $24 \mathrm{~h}$ in serumsupplemented RPMI-1640 medium without PMA. Cell differentiations were verified by evaluating cell adhesion and spreading under an optical microscope. For Fluo-4 AM and ROX epifluorescence imaging, differentiated THP-1 cells are washed three 
times with OPTI-MEM and then incubated with $7.5 \mu \mathrm{M}$ Fluo-4 AM and $6.25 \mu \mathrm{M}$ CellROX Deep Red Reagent in OPTI-MEM for 30 minutes. To remove the free dye thoroughly, the cells are washed with OPTI-MEM three times before imaging.

\section{Macrophage calcium and ROS signal processing}

Data was analyzed in MATLAB (MathWorks) using custom software. Cells were located by tracking local maxima and the intensity over time of a cell-sized disk surrounding the mean position of each cell was analyzed. The code read in a single TIFF file representing a 3D time series. After background subtraction, flat-field correction and Gaussian smoothing, local maxima in each frame were identified. Maxima from each frame were combined into tracks using a nearest-neighbor approach. If the standard deviation of the position was greater than half the cell radius, the track would be discarded. Otherwise, the mean position was assumed to be the location of a cell. The mean intensity of a cell-sized disk around each of these fixed positions was calculated, forming intensity traces for each cell. The noise was reduced by Gaussian smoothing. Sharp increases in calcium intensity were then identified from the first derivative and a user-defined threshold determined which of these were classified as calcium spikes. If a minimum spike intensity and/or minimum spike duration have been specified at the start, spikes not meeting these criteria were discarded. Percentage of ROS signals changed was calculated as

$$
\text { Percent of ROS signals changed }=\frac{F_{\text {final }}-F_{\text {initial }}}{F_{\text {initial }}}
$$

The initial intensity was defined by averaging the DeepRed fluorescence intensities across 50 frames prior to the addition of tau aggregates, and the final intensity was determined by averaging the DeepRed fluorescence intensities across 50 frames prior to the addition of ionomycin. Further details and the source code are available at https://github.com/janehumphrey/calciumStationaryCells. 


\section{TNF- $\alpha$ enzyme-linked immunosorbent assay (ELISA)}

The level of TNF- $\alpha$ secreted into the cell supernatant was measured by Abcam ELISA kit (ab181421) following the manufacture protocol. Total protein concentrations were then determined by Pierce BCA assay following the manufacture protocol to ensure the total protein concentrations were uniform and controlled across different treatment groups. 


\section{References}

1. Kosik, K. S., Joachim, C. L. \& Selkoe, D. J. Microtubule-associated protein tau (T) is a major antigenic component of paired helical filaments in Alzheimer disease. Proc. Nati. Acad. Sci. USA 83, 4044-4048 (1986).

2. Lee, V. M.-Y., Goedert, M. \& Trojanowski, J. Q. Neurodegenerative tauopathies. Annual Review of Neuroscience 24, 1121-1159 (2001).

3. Lim, S., Haque, M. M., Kim, D., Kim, D. J. \& Kim, Y. K. Cell-based models to investigate Tau aggregation. Comput. Struct. Biotechnol. J. 12, 7-13 (2014).

4. Wesseling, H. et al. Tau PTM profiles identify patient heterogeneity and stages of Alzheimer's Disease. Cell 183, 1699-1713.e13 (2020).

5. Wang, Y. \& Mandelkow, E. Tau in physiology and pathology. Nat. Rev. Neurosci. 17, 22 35 (2016).

6. Iqbal, K., Gong, C.-X. \& Liu, F. Hyperphosphorylation-induced tau oligomers. Front. Neurol. 4, 112 (2013).

7. Rankin, C. A., Sun, Q. \& Gamblin, T. C. Pseudo-phosphorylation of tau at Ser202 and Thr205 affects tau filament formation. Mol. Brain Res. 138, 84-93 (2005).

8. Noble, W. et al. Inhibition of glycogen synthase kinase-3 by lithium correlates with reduced tauopathy and degeneration in vivo. Proc. Natl. Acad. Sci. 102, 6990-6995 (2005).

9. Braak, H. \& Braak, E. Staging of Alzheimer's Disease-related neurofibrillary changes. Neurobiol. Aging 16, 271-278 (1995).

10. Hanger, D. P., Anderton, B. H. \& Noble, W. Tau phosphorylation: the therapeutic challenge for neurodegenerative disease. Trends in Molecular Medicine 15, 112-119 (2009).

11. Liu, F. et al. PKA modulates GSK-3 $\beta$ - and cdk5-catalyzed phosphorylation of tau in siteand kinase-specific manners. FEBS Lett. 580, 6269-6274 (2006).

12. Cavallini, A. et al. An unbiased approach to identifying tau kinases that phosphorylate tau at sites associated with alzheimer disease. J. Biol. Chem. 288, 23331-23347 (2013).

13. Yoshida, H. \& Goedert, M. Sequential phosphorylation of tau protein by cAMPdependent protein kinase and SAPK4/p38 $\delta$ or JNK2 in the presence of heparin generates the AT100 epitope. J. Neurochem. 99, 154-164 (2006). 
14. Rankin, C. A., Sun, Q. \& Gamblin, T. C. Tau phosphorylation by GSK-3 $\beta$ promotes tangle-like filament morphology. Mol. Neurodegener. 2, 12 (2007).

15. Šimić, G. et al. Tau protein hyperphosphorylation and aggregation in alzheimer's disease and other tauopathies, and possible neuroprotective strategies. Biomolecules 6, 2-28 (2016).

16. Kopke, E. et al. Microtubule-associated protein tau. Abnormal phosphorylation of a nonpaired helical filament pool in Alzheimer disease. J. Biol. Chem. 268, 24374-24384 (1993).

17. Wegmann, S. et al. Tau protein liquid-liquid phase separation can initiate tau aggregation. EMBO J. 37, e98049 (2018).

18. Despres, C. et al. Major differences between the self-assembly and seeding behavior of heparin-induced and in vitro phosphorylated tau and their modulation by potential inhibitors. ACS Chem. Biol. 14, 1363-1379 (2019).

19. Gamblin, T. C., Berry, R. W. \& Binder, L. I. Modeling tau polymerization in vitro: a review and synthesis. Biochemistry 42, 15009-15017 (2003).

20. Kundel, F. et al. Measurement of tau filament fragmentation provides insights into prionlike spreading. ACS Chem. Neurosci. 9, 1276-1282 (2018).

21. Needham, L. M. et al. ThX-a next-generation probe for the early detection of amyloid aggregates. Chem. Sci. 11, 4578-4583 (2020).

22. Sahara, N. et al. Assembly of two distinct dimers and higher-order oligomers from fulllength tau. Eur. J. Neurosci. 25, 3020-3029 (2007).

23. Scott, C. W., Fieles, A., Sygowski, L. A. \& Caputo, C. B. Aggregation of tau protein by aluminum. Brain Res. 628, 77-84 (1993).

24. Flagmeier, P. et al. Ultrasensitive measurement of $\mathrm{Ca}^{2+}$ influx into lipid vesicles induced by protein aggregates. Angew. Chemie - Int. Ed. 56, 7750-7754 (2017).

25. De, S. et al. Different soluble aggregates of A $\beta 42$ can give rise to cellular toxicity through different mechanisms. Nat. Commun. 10, 1-11 (2019).

26. Varela, J. A. et al. Optical structural analysis of individual $\alpha$-synuclein oligomers. Angew. Chemie - Int. Ed. 57, 4886-4890 (2018).

27. Kundel, F. et al. Hsp70 inhibits the nucleation and elongation of tau and sequesters tau aggregates with high affinity. ACS Chem. Biol. 13, 636-646 (2018). 
28. Drews, A. et al. Inhibiting the $\mathrm{Ca}^{2+}$ influx induced by human CSF. Cell Rep. 21, 33103316 (2017).

29. Ising, C. \& Heneka, M. T. Functional and structural damage of neurons by innate immune mechanisms during neurodegeneration. Cell Death Dis. 9, 120 (2018).

30. Yoshiyama, Y. et al. Synapse loss and microglial activation precede tangles in a P301S tauopathy mouse model. Neuron 53, 337-351 (2007).

31. Nilson, A. N. et al. Tau oligomers associate with inflammation in the brain and retina of tauopathy mice and in neurodegenerative diseases. J. Alzheimer's Dis. 55, 1083-1099 (2017).

32. Schappe, M. S. et al. Chanzyme TRPM7 mediates the $\mathrm{Ca}^{2+}$ influx essential for lipopolysaccharide-induced Toll-like receptor 4 endocytosis and macrophage activation. Immunity 48, 59-74.e5 (2018).

33. Rannikko, E. H., Weber, S. S. \& Kahle, P. J. Exogenous $\alpha$-synuclein induces Toll-like receptor 4 dependent inflammatory responses in astrocytes. BMC Neurosci. 16, 57 (2015).

34. Hughes, C. et al. Beta amyloid aggregates induce sensitised TLR4 signalling causing long-term potentiation deficit and rat neuronal cell death. Commun. Biol. 3, 1-7 (2020).

35. Alonso, A. D. C., Zaidi, T., Novak, M., Grundke-Iqbal, I. \& Iqbal, K. Hyperphosphorylation induces self-assembly of $\tau$ into tangles of paired helical filaments/straight filaments. Proc. Natl. Acad. Sci. U. S. A. 98, 6923-6928 (2001).

36. Goedert, M. et al. Assembly of microtubule-associated protein tau into Alzheimer-like filaments induced by sulphated glycosaminoglycans. Nature 383, 550-553 (1996).

37. Pérez, M., Valpuesta, J. M., Medina, M., Montejo De Garcini, E. \& Avila, J. Polymerization of $\tau$ into filaments in the presence of heparin: The minimal sequence required for $\tau-\tau$ interaction. $J$. Neurochem. 67, 1183-1190 (1996).

38. Zhang, W. et al. Heparin-induced tau filaments are polymorphic and differ from those in Alzheimer's and Pick's diseases. Elife 8, e43584 (2019).

39. Pérez, M., Arrasate, M., Montejo De Garcini, E., Muñoz, V. \& Avila, J. In vitro assembly of tau protein: mapping the regions involved in filament formation. Biochemistry $\mathbf{4 0}$, 5983-5991 (2001).

40. Von Bergen, M. et al. Assembly of $\tau$ protein into Alzheimer paired helical filaments depends on a local sequence motif (306VQIVYK311) forming $\beta$ structure. Proc. Natl. 
Acad. Sci. U. S. A. 97, 5129-5134 (2000).

41. Jeganathan, S., Von Bergen, M., Brutlach, H., Steinhoff, H. J. \& Mandelkow, E. Global hairpin folding of tau in solution. Biochemistry 45, 2283-2293 (2006).

42. Bibow, S. et al. Structural impact of proline-directed pseudophosphorylation at AT8, AT100, and PHF1 epitopes on 441-residue tau. J. Am. Chem. Soc. 133, 15842-15845 (2011).

43. Kunjithapatham, R. et al. Role for the $\alpha$-helix in aberrant protein aggregation. Biochemistry 44, 149-156 (2005).

44. Zhou, Y. et al. Relevance of phosphorylation and truncation of tau to the etiopathogenesis of Alzheimer's disease. Front. Aging Neurosci. 10, 27 (2018).

45. Chen, X., Chen, M., Schafer, N. P. \& Wolynes, P. G. Exploring the interplay between fibrillization and amorphous aggregation channels on the energy landscapes of tau repeat isoforms. Proc. Natl. Acad. Sci. 117, 4125-4130 (2020).

46. Maeda, S. et al. Increased levels of granular tau oligomers: An early sign of brain aging and Alzheimer's disease. Neurosci. Res. 54, 197-201 (2006).

47. Spires-Jones, T. L., Kopeikina, K. J., Koffie, R. M., de Calignon, A. \& Hyman, B. T. Are tangles as toxic as they look? J. Mol. Neurosci. 45, 438-44 (2011).

48. Spires, T. L. et al. Region-specific dissociation of neuronal loss and neurofibrillary pathology in a mouse model of tauopathy. Am. J. Pathol. 168, 1598-1607 (2006).

49. Wu, J. W. et al. Small misfolded tau species are internalized via bulk endocytosis and anterogradely and retrogradely transported in neurons. J. Biol. Chem. 288, 1856-1870 (2013).

50. Jones, E. M. et al. Interaction of tau protein with model lipid membranes induces tau structural compaction and membrane disruption. Biochemistry 51, 2539-2550 (2012).

51. Arispe, N., Pollard, H. B. \& Rojas, E. $\beta$-amyloid $\mathrm{Ca}^{2+}$-channel hypothesis for neuronal death in Alzheimer Disease. Mol. Cell. Biochem. 140, 119-125 (1994).

52. Kim, H. Y. et al. Structural properties of pore-forming oligomers of $\alpha$-synuclein. J. Am. Chem. Soc. 131, 17482-17489 (2009).

53. Kourie, J. I., Farrelly, P. V. \& Henry, C. L. Channel activity of deamidated isoforms of prion protein fragment 106-126 in planar lipid bilayers. J. Neurosci. Res. 66, 214-220 (2001). 
54. Flach, K. et al. Tau oligomers impair artificial membrane integrity and cellular viability. $J$. Biol. Chem. 287, 43223-43233 (2012).

55. Thériault, P., Elali, A. \& Rivest, S. The dynamics of monocytes and microglia in Alzheimer's disease. Alzheimer's Research and Therapy 7, 41 (2015).

56. Alavi Naini, S. M. \& Soussi-Yanicostas, N. Tau hyperphosphorylation and oxidative stress, a critical vicious circle in neurodegenerative tauopathies? Oxid. Med. Cell. Longev. 2015, 1-17 (2015).

57. Bevan-Jones, W. R. et al. Neuroinflammation and protein aggregation co-localize across the frontotemporal dementia spectrum. Brain 143, 1010-1026 (2020).

58. Chen, W. et al. Increased tauopathy drives microglia-mediated clearance of beta-amyloid. Acta Neuropathol. Commun. 4, 63 (2016).

59. Cui, W. et al. Inhibition of TLR4 induces M2 microglial polarization and provides neuroprotection via the NLRP3 inflammasome in Alzheimer's Disease. Front. Neurosci. 14, 444 (2020).

60. Park, B. S. et al. The structural basis of lipopolysaccharide recognition by the TLR4-MD2 complex. Nature 458, 1191-1195 (2009).

61. Marty, M. T. et al. Bayesian deconvolution of mass and ion mobility spectra: From binary interactions to polydisperse ensembles. Anal. Chem. 87, 4370-4376 (2015).

62. Foreman-Mackey, D., Hogg, D. W., Lang, D. \& Goodman, J. emcee : The MCMC Hammer . Publ. Astron. Soc. Pacific 125, 306-312 (2013).

63. Sang, J. C. et al. Direct Observation of Murine Prion Protein Replication in Vitro. J. Am. Chem. Soc. 140, 14789-14798 (2018).

64. Whiten, D. R. et al. Nanoscopic Characterisation of Individual Endogenous Protein Aggregates in Human Neuronal Cells. ChemBioChem 19, 2033-2038 (2018). 


\section{Figures}

a

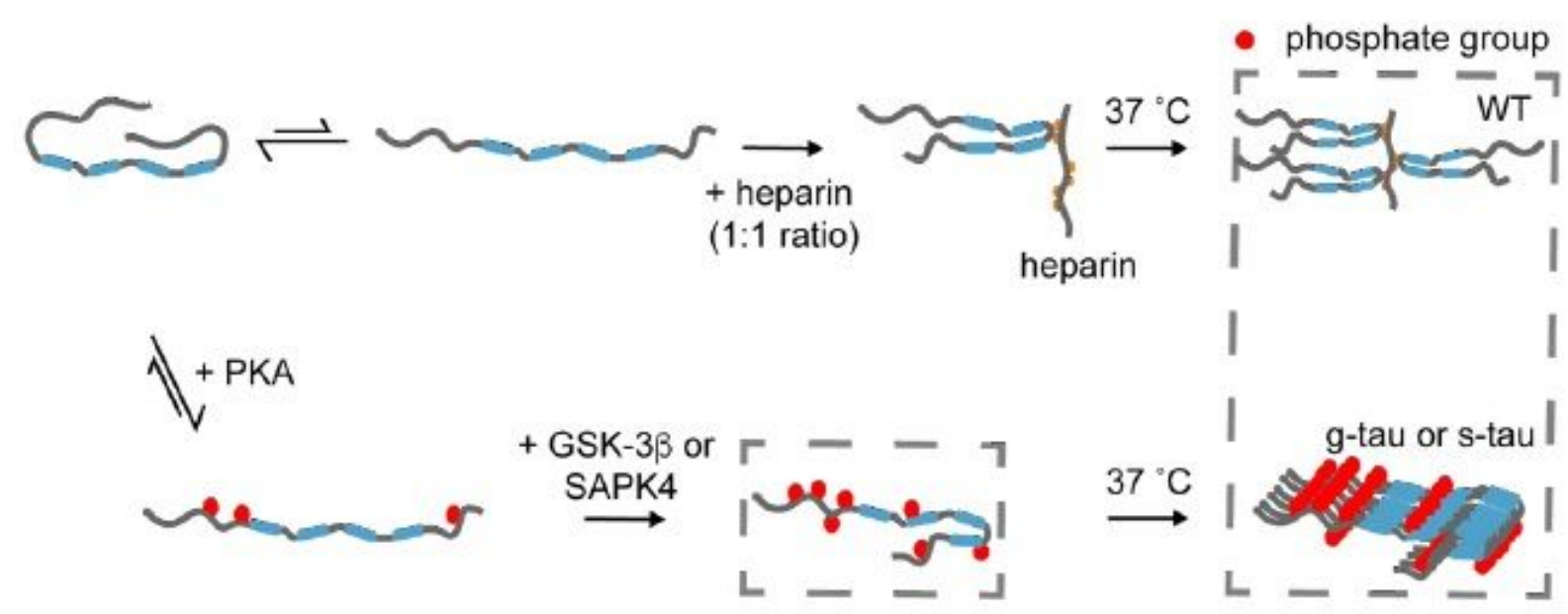

phosphorylation stablizes the opening of the transient folding monomer samples taken for mass spectrometry aggregates taken for structural characterization via fluorescence microscopy b

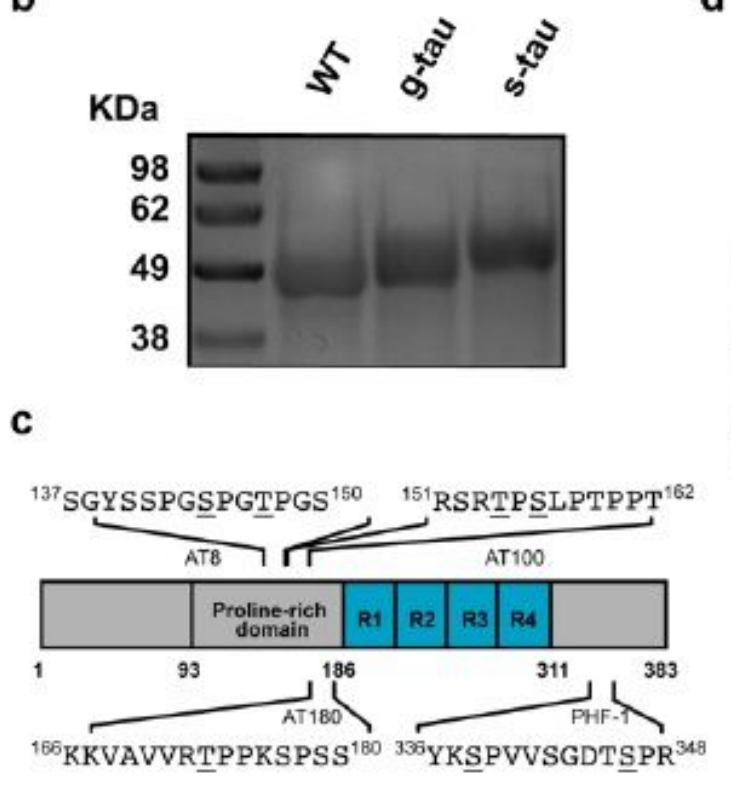

d

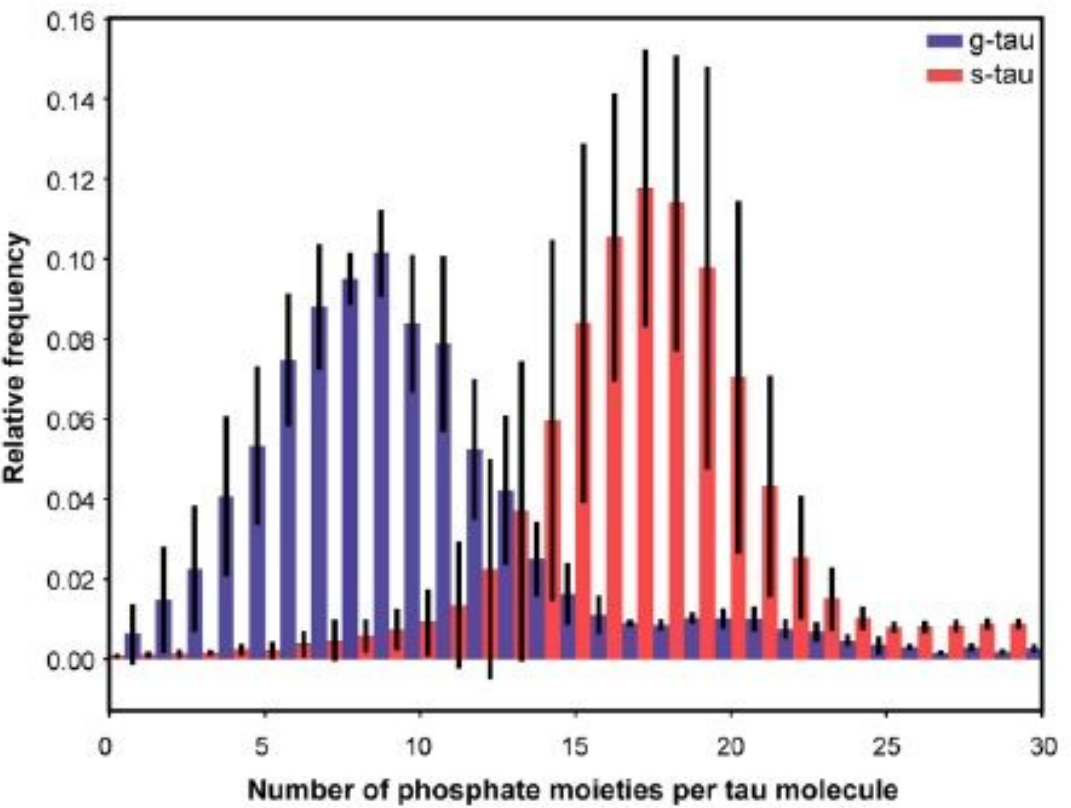

\section{Figure 1}

Sequential hyperphosphorylation of tau in vitro generates AD-specific epitopes. a. A pictorial representation of the experimental design: WT tau was sequentially hyperphosphorylated, first by PKA and then by either GSK-3b or SAPK-4 kinase. b. Representative SDS-PAGE (4-12\%, stained by Coomassie blue) showed an upward shift of electrophoretic mobility for both g-tau and s-tau species, indicating successful phosphorylation reaction. c. LC-MS/MS analysis revealed that both g-tau and s-tau tau were hyperphosphorylated at ADspecific epitopes, such as targeting sites of AT8, AT100, AT180, and PHF-1, highlighted along with the pictorial representation of WT tau. $d$. High-resolution native mass spectrometry 
results indicated on average there were 9 phosphate groups per g-tau molecule while over 18 phosphate groups per s-tau molecule. Error bars represent \pm s.d. from MCMC analysis.

a

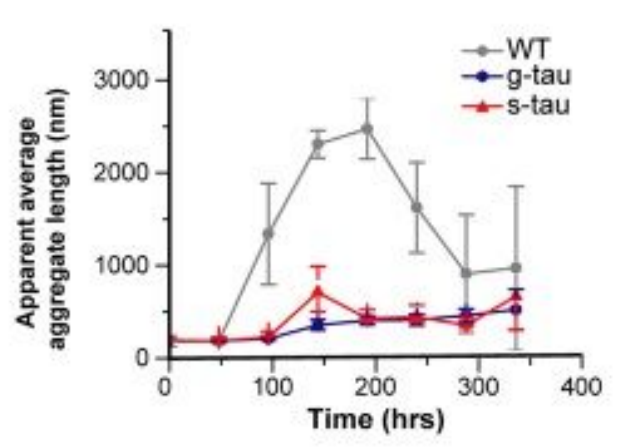

b

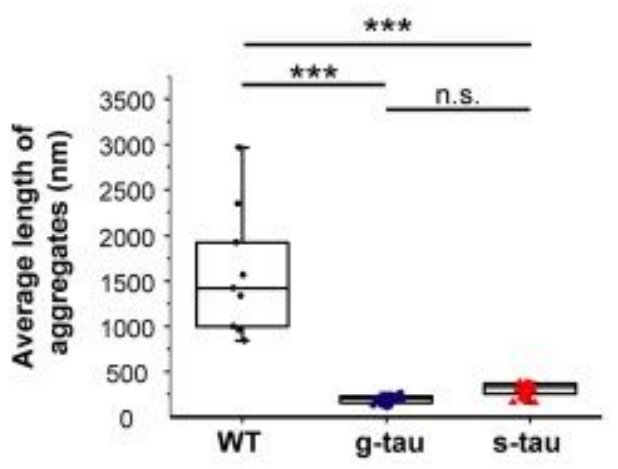

C

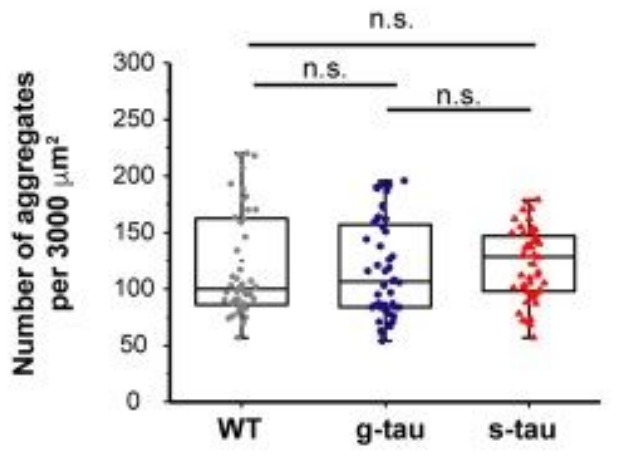

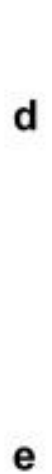
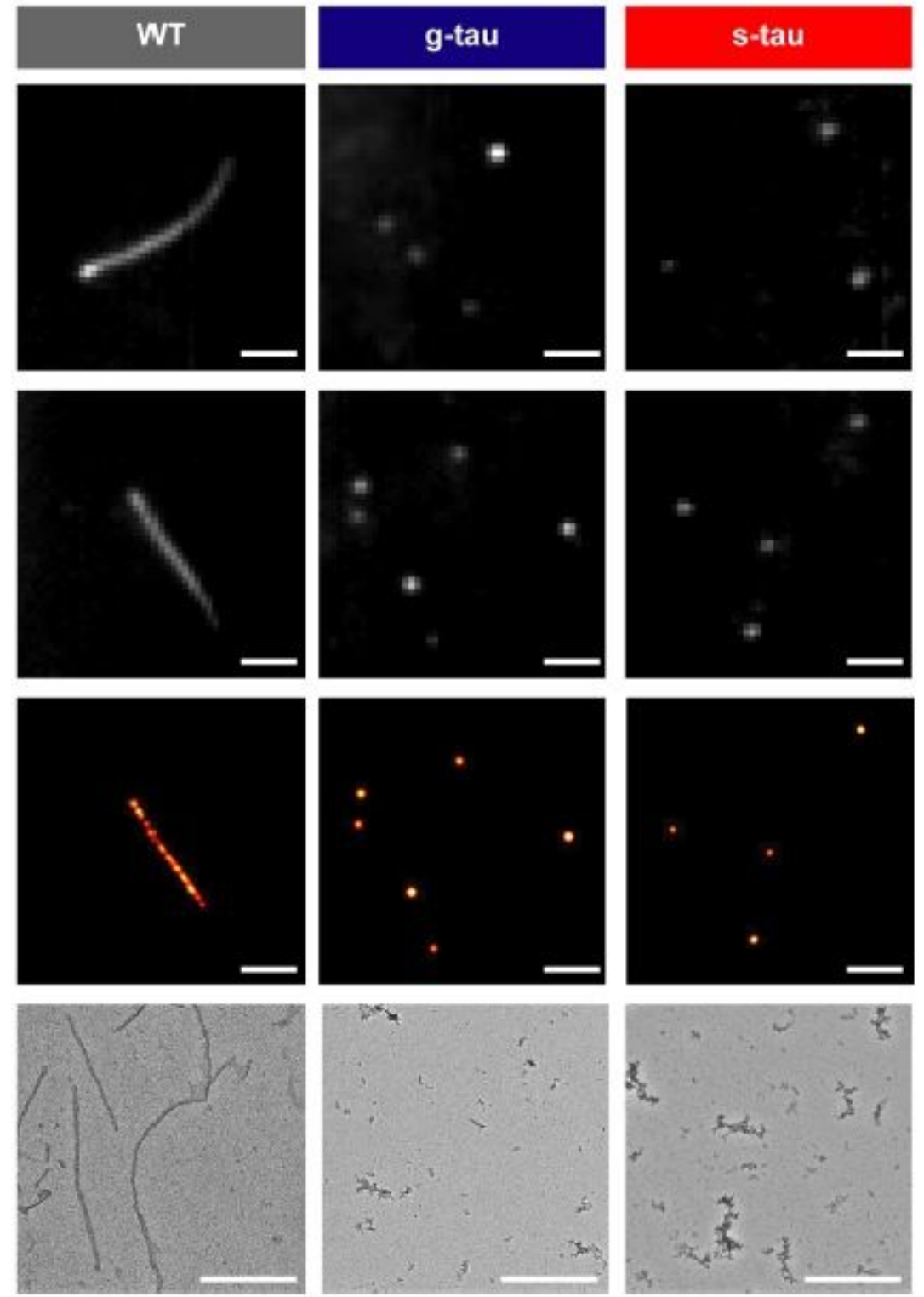

\section{Figure 2}

Hyperphosphorylation enables wildtype tau to self-polymerize into small amorphous aggregates without external inducers. a. The apparent average length of tau aggregates, monitored by fluorescence microscopy, was analyzed and plotted as a function of time (Error bars represent \pm s.d. of $n=3$ ). b. Summary plot based on our results from super-resolution microscopy indicated that at $96 \mathrm{hr}$ of incubation the average length of heparin-induced WT aggregates (1450 $\pm 700 \mathrm{~nm})$ was significantly larger than both g-tau $(190 \pm 50 \mathrm{~nm})$ and or s-tau $(280 \pm 90 \mathrm{~nm})(\mathrm{n}=9$ across three independent experiments for each tau species). c. Summary plot based on our results from fluorescence microscopy indicated the number of aggregates was not significantly different among these three tau species $(n=48$ 
across three independent experiments for each tau species). d. Representative pFTAA diffraction-limited images of different tau species after $96 \mathrm{hr}$ of incubation: while WT tau under the induction of heparin grew into fibrillar aggregates, both g-tau and s-tau self-assembled into small, nonfibrillar aggregates which largely remained as diffraction limited spots. Scale bar: $1.0 \mu \mathrm{m}$. e. Representative ThX stacked and super-resolved images of different tau species after $96 \mathrm{hr}$ of incubation. Scale bar: $1.0 \mu \mathrm{m}$. $\mathrm{f}$.

Morphological examination of all three tau species by TEM after $96 \mathrm{hr}$ of incubation. Scale bar: $500 \mathrm{~nm}$. For $b$ and $c$, the $P$ values are based on unpaired Student's $t$ test: ${ }^{\star} * \star p<0.001 ; n . s .$, nonsignificant.

a
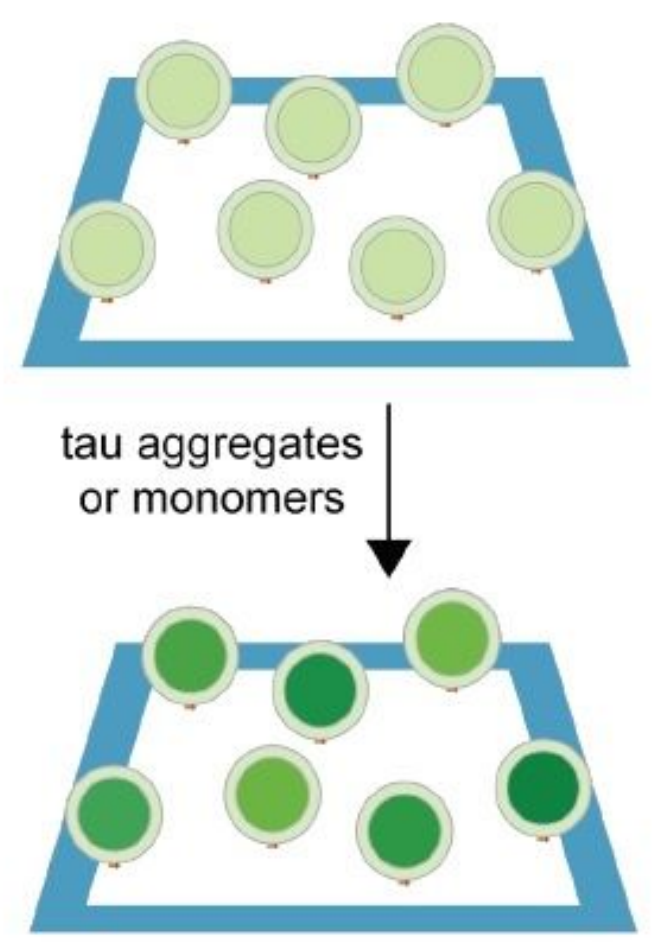

b

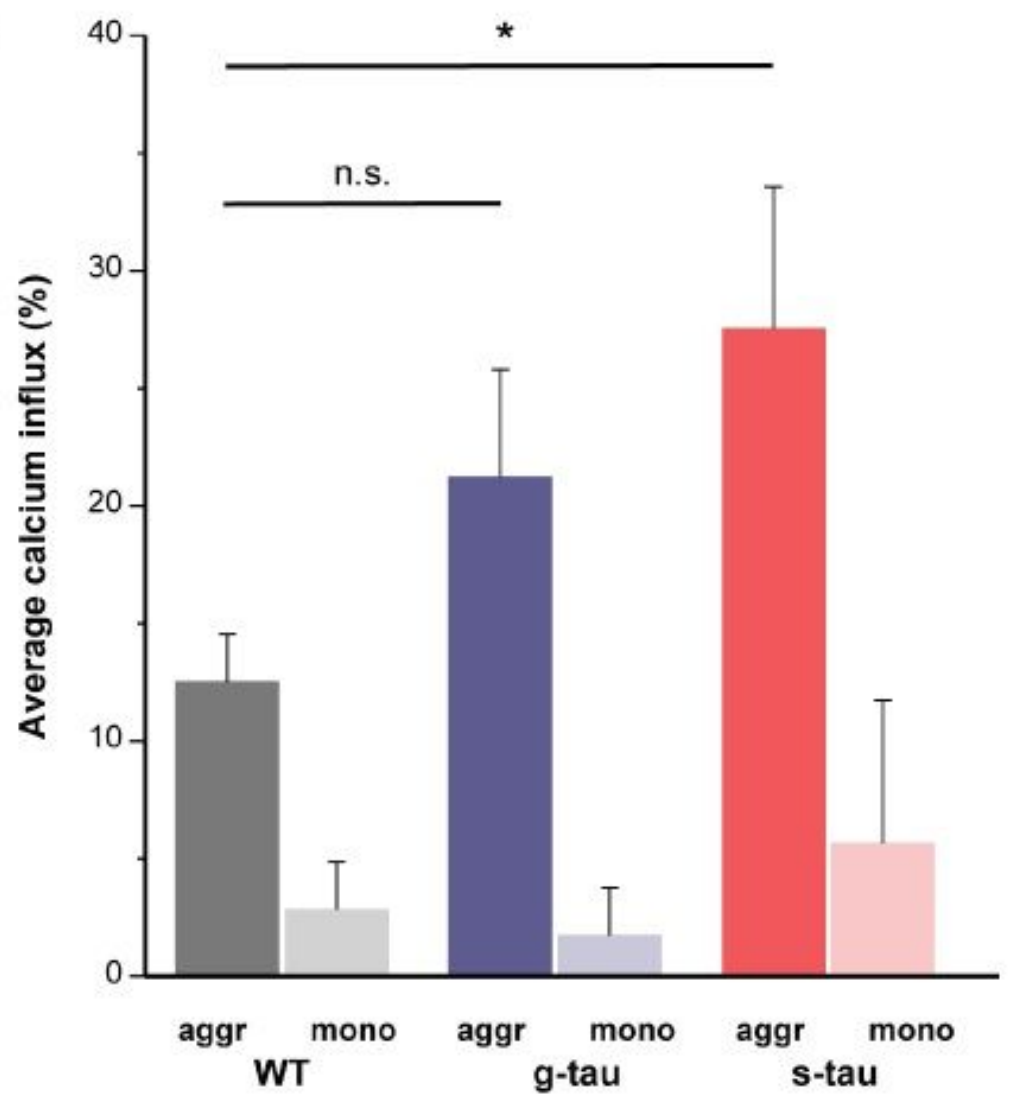

\section{Figure 3}

Hyperphosphorylated tau amorphous aggregates disrupt membrane integrity more effectively than WT fibrillar aggregates. a. The ability to disrupt membrane integrity, an important aspect that explains the toxicity of protein aggregates, was quantified by calcium influx caused by each tau species. The percentage of $\mathrm{Ca} 2+$ influx was normalized by the maximum $\mathrm{Ca} 2+$ influx induced by ionomycin. b. Our results from the liposomal assay revealed that WT tau aggregates caused $12 \pm 2 \%$ calcium influx, g-tau aggregates elicited $21 \pm 5 \%$ calcium influx, and s-tau aggregates induced $28 \pm 6 \%$ calcium influx. All of the monomeric controls caused less calcium influx than their corresponding aggregate samples. So s-tau aggregates can better permeabilize synthetic lipid bilayer than WT aggregates, suggesting a structurefunction relationship of the hyperphosphorylated tau (Error bars represent \pm s.d. of $n=3$ for each tau species, the $P$ values are based on unpaired Student's $t$ test: ${ }^{*} p<0.05$, n.s., nonsignificant). 


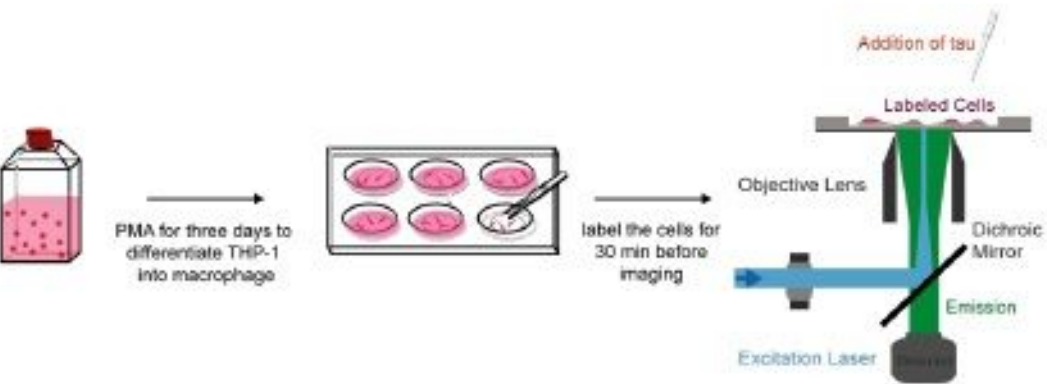

b

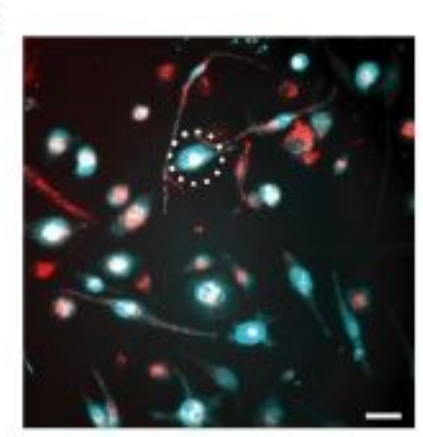

c

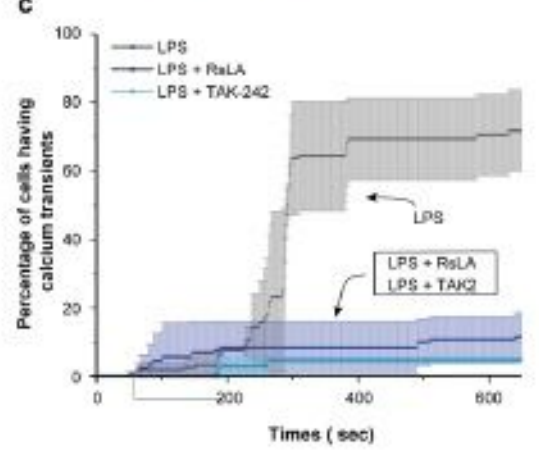

e

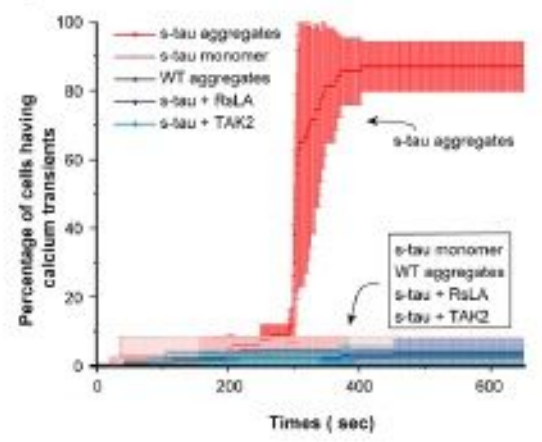

ii

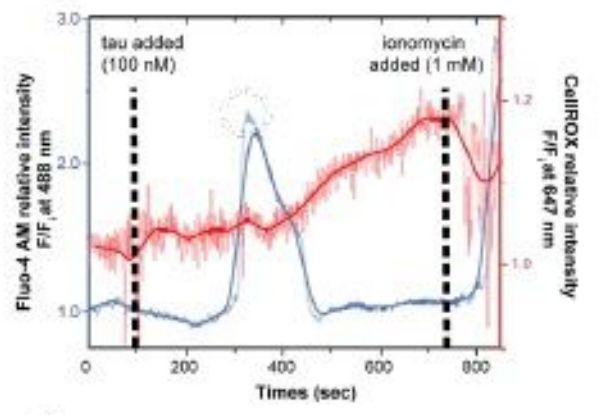

d

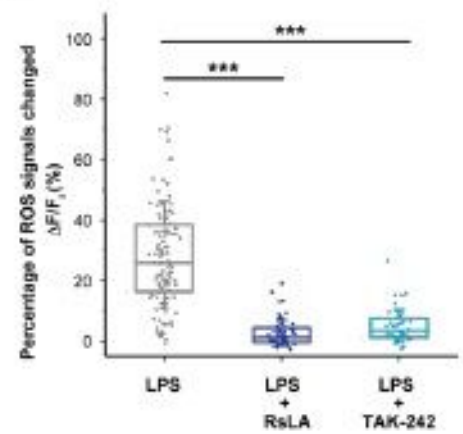

f

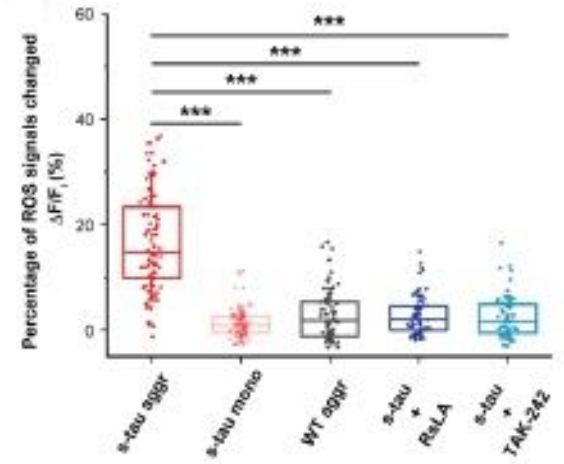

\section{Figure 4}

Hyperphosphorylated tau aggregates elicited calcium transient and stimulated ROS production in human macrophages in a TLR-4 dependent manner. a. A pictorial representation of the macrophage assay workflow. The cytosolic calcium and ROS level were simultaneously monitored as different tau aggregates were applied. b. Representative image of dual-labeled macrophage (i) and calcium and ROS traces (ii) after the treatment of s-tau aggregates. Scale bar: $25.0 \mu \mathrm{m}$. c-d. Summary plots of calcium 
transients (c) and ROX production (d) induced by LPS $(\mathrm{n}=123)$. When treating the cells with RsLA $(\mathrm{n}=$ $70, F(1,4)=40.78)$ or TAK-242 $(n=47, F(1,4)=84.62)$, the LPS-induced calcium transients and ROS level elevation were significantly moderated. e-f. Summary plots of calcium transients (e) and ROX production (f) induced by different tau species and treatment conditions. Compared to WT tau aggregates $(n=63)$, stau aggregates induced strong calcium transients and ROS production $(n=83, F(1,4)=174.88)$. Since stau monomer control did not elicit significant response $(n=89 F(1,4)=110.14)$, ATP and LPS contamination were controlled. The involvement of TLR4 was tested: by pre-treating the cells with RsLA $(n=65, F(1,4)=184.60)$ or TAK-242 $(n=62, F(1,4)=170.11)$, s-tau can no longer elicit significant response. For $c$ and $d$, the $P$ values are based on two-way mixed ANOVA: all $p \otimes 0.001$ except the one between LPS and RsLA which is $p \otimes 0.01$. For $d$ and $f$, the $P$ values are based on unpaired Student's $t$ test: $\star \star \star x p<0.001$. Error bars in $c$ and e represent \pm s.d. of three independent experiments.

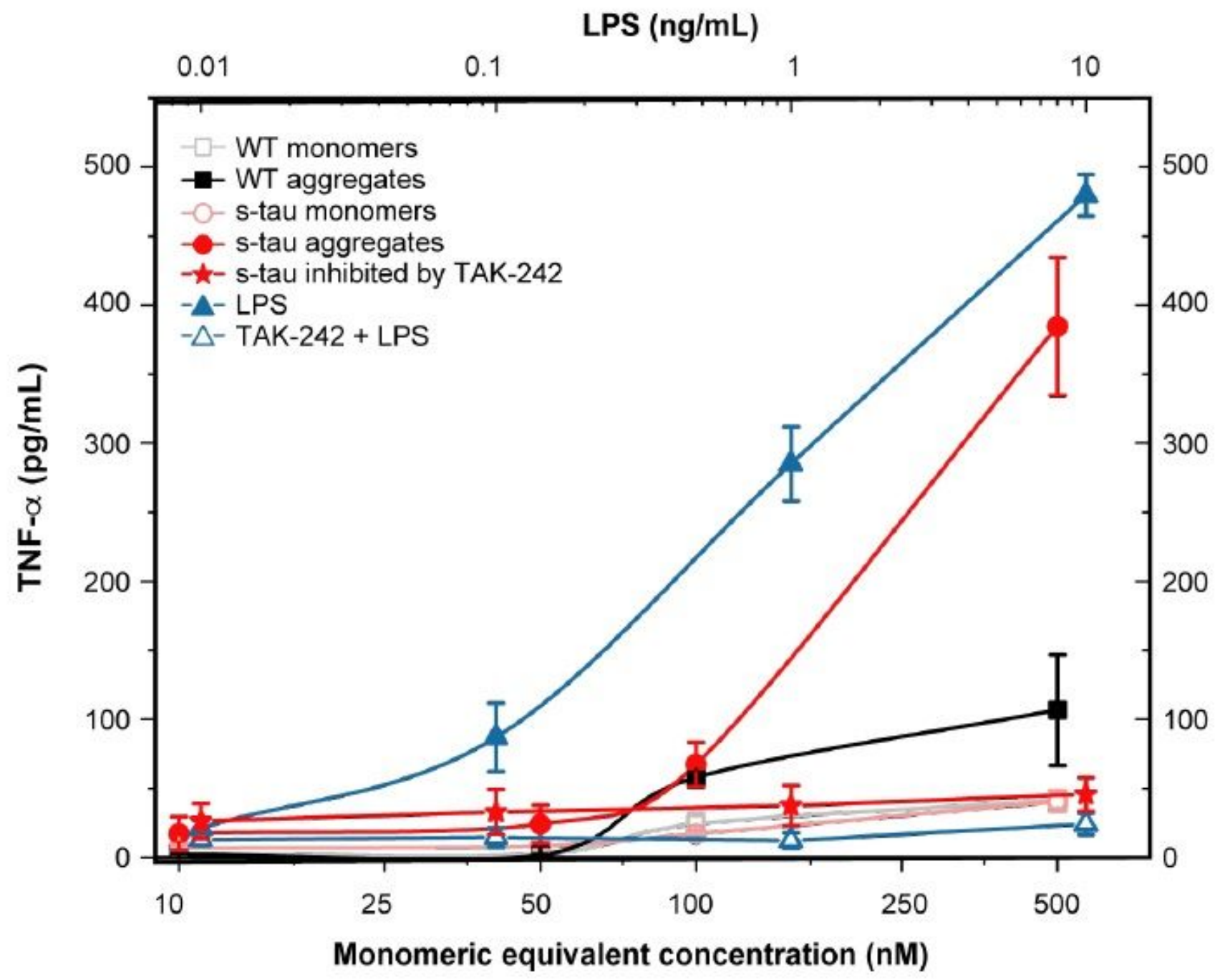

Figure 5

Hyperphosphorylated tau elicited TLR4-mediated inflammation in human macrophages. As a positive control, various concentrations of LPS (varying from $10 \mathrm{pg} / \mathrm{mL}$ to $10 \mathrm{ng} / \mathrm{mL}$ ) were applied to elicit robust 
TLR4 activation, resulting in the release of TNF-a in a dose-dependent fashion. Our results also showed stau aggregates can cause more potent proinflammatory response than WT aggregates after 24 hours of incubation $(F(1,7)=67.07)$. Since monomer controls did not elicit any significant response in comparison with their corresponding aggregate samples $(F(1,7)=16.59$ between WT aggregates and monomers, $F(1,7)=167.46$ between s-tau aggregates monomers), ATP and LPS contamination were controlled. The involvement of TLR4 was subsequently tested: the inhibitory effect of TAK242 was first being verified since the inhibitor successfully suppressed the level of TNF-a $(F(1,7)=1120.74)$. When inhibited by TAK242 , the s-tau can no longer elicit any significant inflammatory response, thereby suggesting the observed inflammation caused by tau aggregated was mediated by TLR4. The P values are based on one-way measures ANOVA: error bars represent \pm s.d. of $n=4$ independent experiments for each condition and all $p<0.001$. 